\title{
Benthic macrofaunal bioturbation activities from shelf to deep basin in spring to summer transition in the Arctic Ocean
}

\author{
Oleszczuk Barbara ${ }^{1, *}$, Michaud Emma ${ }^{2}$, Morata Nathalie ${ }^{2,3}$, Renaud Paul E. ${ }^{3,4}$, Kędra Monika ${ }^{1}$
}

1 Institute of Oceanology, Polish Academy of Science (IOPAN), Powstańców Warszawy 55, 81-712,

Sopot, Poland

2 Laboratoire des Sciences de L'environnement Marin Sciences (LEMAR), UMR 6539 (CNRS/UBO/

IRD/Ifremer), Institut Universitaire Européen de la Mer, rue Dumont d'Urville, 29280, Plouzané, France

${ }^{3}$ Akvaplan-niva, Fram Centre for Climate and the Environment, Troms $\varnothing$, Norway

4 The University Centre in Svalbard, Longyearbyen, Norway

* Corresponding author : Barbara Oleszczuk, email address : oleszczuk@iopan.gda.pl

\begin{abstract}
:
The aim of this study was to assess bioturbation rates in relation to macrozoobenthos and environmental variables in the Svalbard fjords, Barents Sea and Nansen Basin during spring to summer transition. The results showed differences in benthic community structure across sampled area in relation to sediment type and phytopigment content. Fjords, Barents Sea and the shallow parts of Nansen Basin $(<400 \mathrm{~m})$ were characterized by high functional groups diversity, and by biodiffusive and non-local rates ranging from 0.05 to $1.75 \mathrm{~cm}-2 \mathrm{y}-1$ and from 0.2 to $3.2 y-1$, respectively. The deeper parts of Nansen Basin, dominated by conveyors species, showed only non-local transport rates $(0.1-1 \mathrm{y}-1)$. Both coefficients intensity varied with benthic biomass. Nonlocal transport increased with species richness and density and at stations with mud enriched by fresh phytopigments, whereas biodiffusion varied with sediment type and organic matter quantity. This study quantified for the first time the two modes of sediment mixing in the Arctic, each of which being driven by different environmental and biological situations.
\end{abstract}

\section{Highlights}

- This is the first complex report on bioturbation in spring to summer transition conducted over a large depth gradient in the Arctic Ocean. - Benthic community structure and related biodiffusion and non-local transport varied in Svalbard fjords, Barents Sea and Nansen Basin. Changes in environmental conditions, and related changes in quality and quantity of available organic matter, had impact on benthic communities and bioturbation. L Large inputs of fresh OM to the seabed can trigger bioturbation activities.

Keywords : non-local transport, biodiffusive transport, macrozoobenthos, spring season, sea ice cover, Arctic Ocean 
46 The structure and functioning of benthic communities depend on the quality and quantity 47 of organic matter $(\mathrm{OM})$ export fluxes to the sea floor and this dependence increases with 48 increasing depth. Shallow Arctic shelves benthos is often fueled by high OM fluxes to the sea 49 floor due to tight pelagic-benthic coupling (e.g. Grebmeier et al., 2006; Tamelander et al., 50 2008), while deep-sea communities become food-limited due to low amount of OM reaching 
sea floor (Maiti et al., 2010). The seasons strongly shape the OM fluxes to the sea floor in the Arctic marine ecosystems. Phytoplankton and ice algae are two principal sources of primary production (PP) in the Arctic Ocean with ice algae being the first food source available after polar night (Søreide et al., 2006, 2008; Leu et al., 2010). Although phytoplankton is quantitatively dominant, ice algal blooms tend to occur earlier in the seasonally ice-covered Arctic seas and may contribute up to 50-60\% of total PP (Gosselin et al., 1997; McMinn et al., 2010; Fernandez-Mendez et al., 2015; Van Leeuwe et al., 2018). During the spring, PP is typically greater than zooplankton consumption and thus highest vertical carbon fluxes are recorded (Andreassen and Wassmann, 1998; Tamelander et al., 2006). Later in the season, the zooplankton grazing reduces the OM flux but also adds to it by producing fecal pellets, which helps phytoplankton sink rapidly to the sea bottom (Olli et al., 2002). In fjords and on the shelf, benthic communities can also be fueled by terrestrial OM carried by rivers and/or glaciers, mainly during summer (Bourgeois et al., 2016). Benthic organisms act as temporal couplers in the seasonal systems, since they can consume variable carbon sources over the different seasons (McMeans et al., 2015), therefore benthic communities reflect rather long term (months to years) water column production, while the benthic activities reflect short term (days to weeks) environmental conditions (Morata and Renaud, 2008).

Bioturbation occurs when an organism moves through the sediment, constructs and maintains burrows, and ingests and defecates. This process results in mixing of particles and solutes within the substratum (Kristensen et al., 2012), and alters sediment structure (e.g., grain size distribution; Montserrat et al., 2009), and production, mineralization and redistribution of OM (Kure and Forbes, 1997). Life habit, motility, and manner of feeding of infaunal species induce either random particle movement over a short distance (biodiffusion (Db) hereafter) (Gérino et al., 2007; Meysman et al., 2003) or biologically induced discontinuous particle transfer between the sediment surface and deeper sediment layers, for 
example via burrowing or feeding behavior (non-local transport (r) hereafter) (Boudreau, 1986; Meysman et al., 2003; Duport et al., 2007; Gogina et al., 2017). According to the mode of particle mixing, benthic organisms can be classified into five functional groups of sediment reworking which may include biodiffusion and/or non-local transport: biodiffusors, gallerydiffusors, upward- and downward-conveyors, and regenerators (François et al., 1997). The presence and intensity of these bioturbation modes are therefore mediated by fauna characteristics like biomass, density, burrowing depth or feeding behavior (François et al., 1999; Gérino et al., 1998; Sandnes et al., 2000; Gilbert et al., 2007; Michaud et al., 2005, 2006; Duport et al., 2007; Aschenbroich et al., 2017). In turn, species composition, nature and intensity of their effects on sediment mixing depends on temperature (Ouelette et al., 2004; Duport et al., 2007; Maire et al., 2007), food inputs (Nogaro et al., 2008) and sediment characteristics (Needham et al., 2011). Changes in species composition and activities, and therefore in bioturbation mode and/or intensity, are expected to influence biochemical processes near the sediment-water interface, including carbon cycling. Bioturbation rate can therefore be influenced by seasonal changes in PP in the above water column and deposited OM in the seafloor (food bank; Morata et al., 2015).

Only a few studies of bioturbation exist in the Arctic Ocean. Teal et al. (2008) created the database with global bioturbation intensity coefficient $(\mathrm{Db})$ and layer depth $(\mathrm{L})$, where they showed that the Arctic, Central Pacific and most tropical regions are missing bioturbation data. In polar regions, it has been shown that sediment mixing rates were higher through biological transports in the shallow sediments directly impacted by the OM input along the marginal ice covered area of the Barents Sea (Maiti et al., 2010) and in the Svalbard fjords (Konovalov et al., 2010). On the contrary, the deep sediments of the Arctic Ocean were marked by lower sediment mixing rates in relation to a lower benthic biomass correlated with lower OM inputs (Clough et al., 1997). Soltwedel et al. (2019), however, did not confirm a 
101 higher bioturbation activity in the high productive Marginal Ice Zone (MIZ) in Fram Strait

102 compared to the less productive ice zone. Seasonal aspects of bioturbation in the Arctic were

103 preliminarily studied by Morata et al. (2015), whose experiments showed that the bioturbation

104 activity was positively correlated with fresh food input during the polar night. McClintic et al.

105 (2008) found no seasonal variation in bioturbation intensity during June and October in West

106 Antarctic continental shelf which suggests that deposit feeders are able to access food

107 particles accumulated during high PP periods. Still, our knowledge on benthic communities

108 responsible for bioturbation processes and their relation to OM inputs in the Arctic Ocean and

109 adjacent shelves remains limited, particularly during the spring bloom.

110 The main aim of this study was to understand the impacts of differences in

111 environmental conditions on benthic communities and their bioturbation function during the

112 spring to summer transition. We focused on the Svalbard area where fjords, shelf and deep

113 Nansen Basin differ considerably in terms of physical forcing affecting the quality and

114 quantity of the OM inputs to the seafloor. Sediment reworking rates were quantified in

115 relation to taxonomic and functional composition of the benthic macrofaunal communities,

116 and in relation to the environmental variables. This work is the first study on bioturbation

117 processes conducted in the Arctic Ocean during spring to summer transition time over a large

118 depth gradient. It will contribute to our understanding of response of macrofauna and their

119 activity to the quality and quantity of $\mathrm{OM}$ in the Arctic seabed.

120

121 2. Material and methods

122

123

2.1. Study area

124 
Sampling was conducted in the Svalbard Archipelago, the Barents Sea and deep Nansen

Basin north of Svalbard (Fig. 1, Table 1). This area is highly influenced by cold Arctic Water coming from the north and warm Atlantic Waters coming from the south, and the relative

128 influence of those two water masses varies largely in the study area.

129

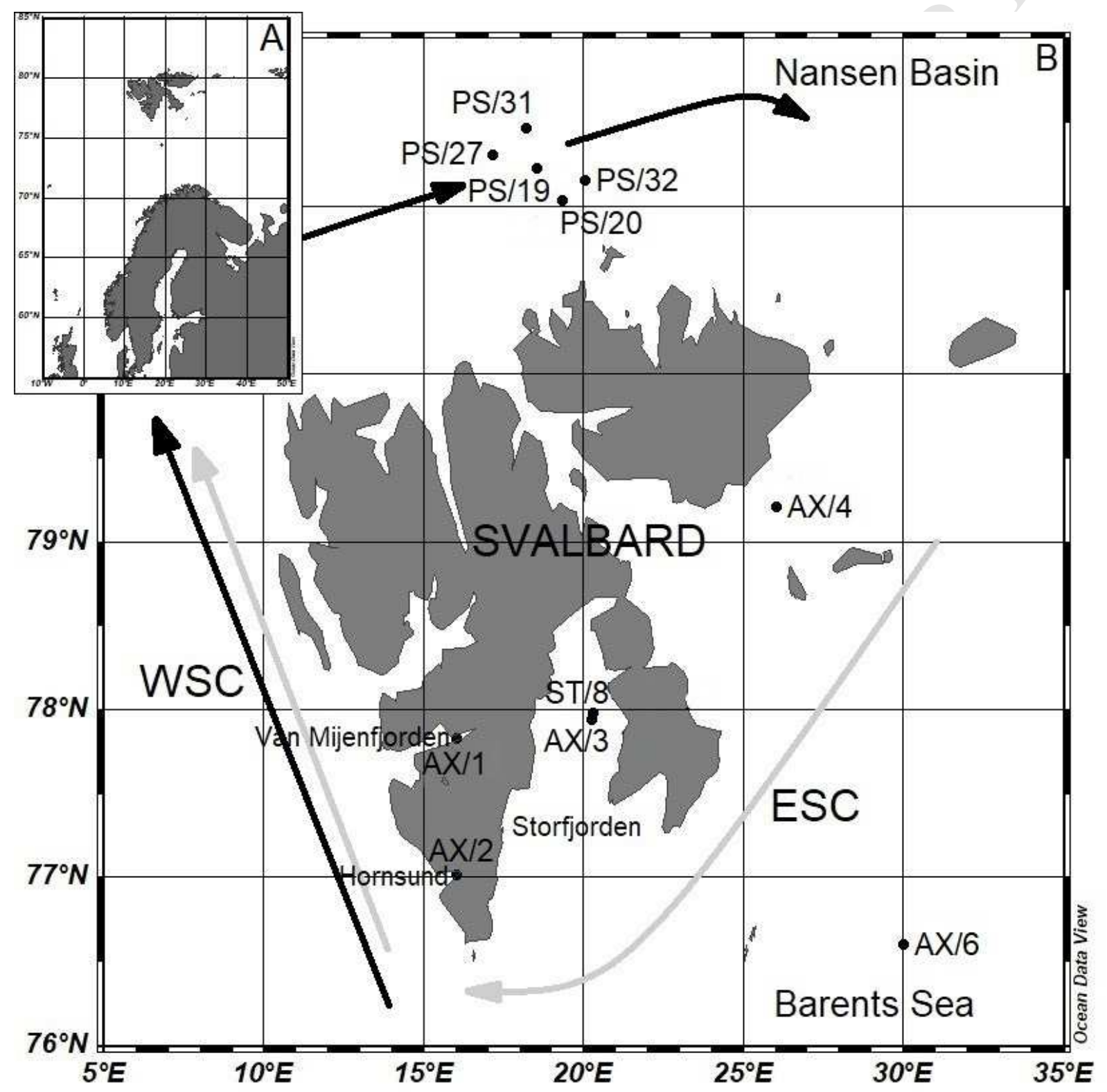

131 Fig. 1. Geographical location of the study region (A) and (B) sampling locations during two

132 cruises (AX - ARCEx, PS - TRANSSIZ) with two major currents surrounding Svalbard: 
133 WSC - West Spitsbergen Current, warm Atlantic waters (black) and the ESC - East

134 Spitsbergen Current, cold Arctic waters (gray) (after Svendsen et al., 2002). 
Table 1. Main characteristics of the sampling stations.

\begin{tabular}{|c|c|c|c|c|c|c|c|c|c|c|}
\hline Station & Date & Cruise name & $\begin{array}{l}\text { No of } \\
\text { cores }\end{array}$ & Area & $\begin{array}{l}\text { Latitude } \\
\left({ }^{\circ} \mathbf{N}\right)\end{array}$ & $\begin{array}{l}\text { Longitude } \\
\quad\left({ }^{\circ} \mathbf{E}\right)\end{array}$ & $\begin{array}{l}\text { Main } \\
\text { current }\end{array}$ & $\begin{array}{l}\text { Depth } \\
{[\mathrm{m}]}\end{array}$ & $\begin{array}{c}\text { Bottom Water } \\
\text { Salinity }\end{array}$ & $\begin{array}{c}\text { Bottom Water } \\
\text { Temperature } \\
\left({ }^{\circ} \mathrm{C}\right) \\
\end{array}$ \\
\hline $\mathbf{A X} / \mathbf{1}$ & 19.05.2016 & ARCEx & 5 & Van Mijenfjorden & $77.83^{\circ}$ & $16.47^{\circ}$ & ESC & 59 & 34.5 & -0.8 \\
\hline $\mathrm{AX} / 2$ & 20.05 .2016 & ARCEx & 5 & Hornsund & $77.02^{\circ}$ & $16.45^{\circ}$ & $\mathrm{ESC}$ & 121 & 34.5 & -0.8 \\
\hline $\mathbf{A X} / \mathbf{3}$ & 21.05 .2016 & ARCEx & 5 & Storfjorden & $77.94^{\circ}$ & $20.22^{\circ}$ & ESC & 96 & 34.5 & -0.8 \\
\hline ST/8 & 15.07.2016 & SteP & 4 & Storfjorden & $77.98^{\circ}$ & $20.28^{\circ}$ & $\mathrm{ESC}$ & 99 & 34.1 & 4.5 \\
\hline $\mathrm{AX} / 4$ & 24.05.2016 & ARCEx & 5 & Erik Eriksen Strait & $79.21^{\circ}$ & $26.00^{\circ}$ & $\mathrm{ESC}$ & 217 & 34.7 & 0.5 \\
\hline $\mathrm{AX} / 6$ & 25.05 .2016 & ARCEx & 5 & Southern Barents Sea & $76.60^{\circ}$ & $30.01^{\circ}$ & ESC & 278 & 35.0 & 2.5 \\
\hline PS/20 & 30.05 .2015 & TRANSSIZ & 3 & Northern Barents Sea & $81.04^{\circ}$ & $19.32^{\circ}$ & WSC & 170 & 34.9 & 0.9 \\
\hline PS/32 & 06.06 .2015 & TRANSSIZ & 4 & Northern Barents Sea & $81.16^{\circ}$ & $20.01^{\circ}$ & WSC & 312 & 34.9 & 2.1 \\
\hline PS/19 & 29.05 .2015 & TRANSSIZ & 5 & Northern Barents Sea & $81.23^{\circ}$ & $18.51^{\circ}$ & WSC & 471 & 35.1 & 1.4 \\
\hline PS/27 & 01.06 .2015 & TRANSSIZ & 5 & Northern Barents Sea & $81.31^{\circ}$ & $17.15^{\circ}$ & WSC & 842 & 34.9 & 0.2 \\
\hline $\mathbf{P S} / \mathbf{3 1}$ & 04.06 .2015 & TRANSSIZ & 5 & Nansen Basin & $81.47^{\circ}$ & $18.17^{\circ}$ & WSC & 1656 & 34.9 & 2.5 \\
\hline
\end{tabular}

136 
Van Mijenfjorden and Hornsund are located on the west coast of Spitsbergen,

141 Svalbard. Van Mijenfjorden is a small fjord, nearly closed by an island at its mouth. It is 142 separated into two basins: the outer (115 $\mathrm{m}$ depth) and inner (74 $\mathrm{m}$ depth), and by $45 \mathrm{~m}$ deep

143 sill that restricts exchange of water between the fjord and the coastal waters (Skarđhamar and

144 Svendsen, 2010). Hornsund is a large open glacial fjord with eight major tidal glaciers located

145 in the central and inner parts and large terrestrial inflow (Błaszczyk et al., 2013; Drewnik et

146 al., 2016). The average depth is $90 \mathrm{~m}$ with a maximum of $260 \mathrm{~m}$ (Kędra et al., 2013). Strong

147 gradients in sedimentation, PP and benthic fauna occur along the increasing distance to the

148 glaciers (Włodarska-Kowalczuk et al., 2013). These high latitude fjords are productive

149 systems, where PP starts in early spring and continue to late autumn (Fetzer et al., 2002). The

150 annual PP reaches up to $216 \mathrm{~g} \mathrm{C} \mathrm{m}^{-2} \mathrm{y}^{-1}$ in Hornsund (Smoła et al., 2017). The Barents Sea is

151 a shelf sea with water depths ranging from $35 \mathrm{~m}$ in the Svalbard Bank to up to $400 \mathrm{~m}$ or more

152 in deep depressions and proximal canyon boundaries (Cochrane et al., 2012). The southern

153 part of the Barents Sea is relatively warm and ice free while its northern parts are seasonally

154 ice covered, with maximum ice coverage from March to April and minimum ice coverage

155 generally occurring in September (Vinje, 2009; Ozhigin et al., 2011; Jørgensen et al., 2015). It

156 is one of the most productive areas in the Arctic Ocean with average PP about $100 \mathrm{~g} \mathrm{C} \mathrm{m}^{-2} \mathrm{y}^{-1}$

157 and maximum PP reaching over $300 \mathrm{~g} \mathrm{C} \mathrm{m}^{-2} \mathrm{y}^{-1}$ on shallow banks (Sakshaug, 2004).

158 Storfjorden is located east of Spitsbergen and has a maximum depth of $190 \mathrm{~m}$ (Skogseth et al.,

159 2005). A polynya appears regularly in Storfjorden. It is a very productive area of the Barents

160 Sea, and its productivity is correlated with the duration of the seasonal sea cover

161 (Winkelmann and Knies, 2005). In Storfjorden the production of marine organic carbon may

162 exceed $300 \mathrm{mg} \mathrm{C} \mathrm{cm}^{-2} \mathrm{kyr}^{-1}$, while the production of total organic carbon (TOC) may exceed

$163500 \mathrm{mg} \mathrm{C} \mathrm{cm}^{-2} \mathrm{kyr}^{-1}$ (Pathirana et al., 2013; Rasmussen and Thomsen, 2014). Nansen Basin, 
164 with a maximum depth of $4000 \mathrm{~m}$, is part of the Eurasian basin of the Arctic Ocean. In

165 general, annual gross $\mathrm{PP}$ is within the range of 5-30 $\mathrm{g} \mathrm{C} \mathrm{m}^{-2}$ (Codispoti et al., 2013).

\subsection{Sampling}

Benthic sampling was conducted during spring cruises of R/V Polarstern PS92 -

TRANSSIZ in May and June 2015, and R/V Helmer Hanssen - ARCEx in May 2016 (Table

1). Samples were collected at 10 stations located along the depth gradient, from Svalbard PS/19, PS/27 and PS/31) were sea ice covered during sampling, except PS/20 station. One station in Storfjorden (AX/3) was revisited in July 2016 during the cruise of R/V L'Atalante STeP $2016(\mathrm{ST} / 8)$.

At each station the bottom water temperature and salinity were determined by the shipboard Conductivity Temperature Density (CTD) rosette. Bottom-water samples were

179 collected using Niskin bottles attached to a CTD and were filtered on pre-combusted

180 Whatman GF/F glass microfiber filters in triplicate and frozen at $-20{ }^{\circ} \mathrm{C}$ for later analyses of

181 bottom water organic carbon $\left(\mathrm{BW} \mathrm{C}_{\text {org }}\right.$ ), total nitrogen $\left(\mathrm{BW} \mathrm{N}_{\text {tot }}\right), \delta^{13} \mathrm{C}\left(\mathrm{BW} \delta^{13} \mathrm{C}\right), \delta^{15} \mathrm{~N}(\mathrm{BW}$ $\left.\delta^{15} \mathrm{~N}\right)$, and $\mathrm{C} / \mathrm{N}$ ratio $(\mathrm{BW} \mathrm{C} / \mathrm{N})$.

184 overlying water from box corer was gently removed from sediment surface and push-cores 185 samples (12 $\mathrm{cm} \varnothing$ and $20 \mathrm{~cm}$ deep, $113.0940 \mathrm{~cm}^{2}$ surface layer) were collected. The top $2 \mathrm{~cm}$ 186 sediment of the core was sampled for biogeochemical variables (grain size, chlorophyll $a$ (Chl 187 a) and phaeopigments (Phaeo), organic matter (SOM), organic carbon (Sed $\mathrm{C}_{\text {org }}$ ) and total 
nitrogen $\left(\right.$ Sed $\left.\mathrm{N}_{\mathrm{tot}}\right)$ ). Samples were frozen in $-20{ }^{\circ} \mathrm{C}$ and transported to the laboratory for analysis.

Table 1) were kept in dark cold room on board (i.e., temperature at $2{ }^{\circ} \mathrm{C}$, the average between $-0.8^{\circ} \mathrm{C}$ and $4.5^{\circ} \mathrm{C}$ being the range of temperatures observed in the bottom waters, Table 1$)$. disturbing the resident infauna. Cores were then filled with bottom water and aerated by bubbling to keep the overlying water saturated with oxygen. Overlying water was renewed every four days. Sediment cores were incubated in those conditions for 10 days which is the minimum time to enable the characterization of the different transport modes. Incubation time that exceeds 15 days increases the probability of complete homogenization of the sedimentary column, and may thus prevent the differentiation of transport modes (François et al., 1997).

This choice of 10 days for duration of experiment was a compromise between the response that we were expecting from the benthic communities and the available time on board to process the experiments.

After this time of incubation in stable conditions the surface water was carefully removed and cores were sliced horizontally in $0.5 \mathrm{~cm}$ layers from 0 to $2 \mathrm{~cm}$ depth, and in 1 $\mathrm{cm}$ layers between 2 and $10 \mathrm{~cm}$ depth. In total, 12 samples were taken, and each sediment 208 layer was homogenized. A subsample of each sediment layer was directly frozen $\left(-20^{\circ} \mathrm{C}\right)$ and used for bioturbation analyses. The remaining sediment of each core samples were sieved

211 buffered formaldehyde. 

sieved into coarse-grained fractions $(>0.250 \mathrm{~mm})$ and fine-grained $(<0.250 \mathrm{~mm})$. For the fine fraction, analyses were performed using a Malvern Mastersizer 2000 laser particle analyzer and presented as volume percent. Mean grain size parameters were calculated using the geometric method of moments in the program GRADISTAT 8.0 (Blott and Pye, 2001).

Pigment concentrations were analyzed fluorometrically following methods described in Holm-Hansen et al. (1965) to determine Chl $a$ and Phaeo concentrations. About $1 \mathrm{~g}$ of dried sediment was extracted with $10 \mathrm{ml}$ of $90 \%$ acetone at $4{ }^{\circ} \mathrm{C}$ in the dark. After $24 \mathrm{~h}$, sediment was then centrifuged (3000 rpm for $2 \mathrm{~min}$ ), and analysed using a Turner Designs AU-10 fluorometer before and after acidification with $100 \mu \mathrm{l} 0.3 \mathrm{M} \mathrm{HCl}$.

For sediment and bottom water biogeochemical parameters analyses, sediments and filters were dried, homogenized and weighed into silver capsules. For sediment and bottom water $\delta^{13} \mathrm{C}$ and $\delta^{15} \mathrm{~N}, \mathrm{C}_{\text {org }}$ and $\mathrm{N}_{\text {tot }}$ analyses, samples were acidified with $2 \mathrm{M} \mathrm{HCl}$ to remove 228 inorganic carbon and dried at $60^{\circ} \mathrm{C}$ for $24 \mathrm{~h}$. The analyses were performed on an Elemental 229 Analyzer Flash EA 1112 Series combined with an Isotopic Ratio Mass Spectrometer IRMS Delta V Advantage (Thermo Electron Corp., Germany). SOM content was measured as loss on ignition at $450^{\circ} \mathrm{C}$ for $4 \mathrm{~h}$ (Zaborska et al., 2006). Sed $\mathrm{C}_{\text {org }}$ content was measured following

232 the method of Kennedy et al. (2005). About $10 \mathrm{mg}$ of dried sediment was acidified with $50 \mu \mathrm{l}$ 233 of $1 \mathrm{~N} \mathrm{HCl}$ three times. Analyses were run on a Thermo Quest Flash EA $1112 \mathrm{CHN}$ analyzer.

\subsection{Benthic community analysis}


In the laboratory, macrofaunal organisms were picked from sediments under a binocular microscope and identified to the lowest possible taxonomic level. Each taxon was counted, weighed (g wet weight) and transferred to $70 \%$ ethanol. Mobility and feeding (WoRMS Editorial Board, 2019), and burrowing behavior (for references see Table 4) were attributed to each taxon. Benthic fauna was classified into five bioturbation functional groups based on the

242 type of the sediment mixing: biodiffusors, gallery-diffusors, upward- or downward-conveyors, and regenerators. Biodiffusors move particles in a random manner in short distances (Gérino, 1992). Gallery-diffusors transport material from the surface sediment layer to deeper by constructing tubes or tunnels system (François et al., 2002). Upward-conveyors transport material from depth to the sediment surface and downward-conveyors transport sediment nonlocally to deeper layers (Fisher et al., 1980; Knaust and Bromley, 2012). Regenerators create a biodiffusion-like process, with large amounts of sediment transported out of the reworked zone with a strong input to the overlying water column, as well as passive downward transport of surface sediment to the bottom of the burrow after burrow abandonment (Gardner et al., 1987; Knaust and Bromley, 2012). Organism density and biomass were evaluated per taxon, trophic and bioturbation functional group, and in total for each sediment core, and subsequently converted per $1 \mathrm{~m}^{-2}$ (area) in order to provide relevant surface values. The biomass to density (B/D) ratio was calculated per core as a proxy of the mean organism size.

\subsection{Bioturbation analyses}

After the sediment cores were sliced, part of the sediment from each sediment layer was freeze-dried at $-70{ }^{\circ} \mathrm{C}$, and homogenized with a mortar and pestle. Three replicates of 0.2 $260 \mathrm{~g}$ sediment from each layer were taken and placed on a black box $(9.5 \mathrm{~cm} \mathrm{x} 7 \mathrm{~cm})$ under a constant UV light source (350 $\pm 370 \mathrm{~nm}$, Tube UV BLB G5T5 $6 \mathrm{~W})$. Images were taken with 
262 a digital camera (Nikon digital captor 2.342.016 pixels) with $28 \mu \mathrm{m}$ per pixel resolution from

263 a constant $12 \mathrm{~cm}$ from the sediment sample to assure identical acquisition conditions for all

264 images (aperture time $1 \mathrm{~s}$; diaphragm aperture f/13, ISO 200). Images were saved in red-

265 green-blue (RGB) colour in jpeg format. The images were analysed using an image

266 processing toolbox (@mathworks) in order to differentiate luminophores from the background

267 sediment by using an appropriate set of RGB threshold levels (Michaud, 2006). Finally, the

268 particle size appropriate for each luminophore was selected $(6$ pixels $\times 6$ pixels for the

269 smallest luminophores), and the pictures were corrected (cleaned) by removing the particle

270 sizes smaller and larger than the actual size of the specific luminophore $(90-120 \mu \mathrm{m})$. The

271 sum of areas (in pixels) of the remaining objects and the number of objects (i.e.,

272 luminophores) were calculated for each picture and averaged between the three pictures from

273 each sediment layer. Finally, with these abundances for all sediment depths for each core, the

274 results were computed as the percentage of detected pixels per depth according to the total

275 number of pixels detected per core thus representing the luminophores distribution over depth

276 for each sediment core.

277 The reaction diffusion type model used in this paper to describe luminophore

278 redistribution following macrofaunal reworking is based on the general diagenetic equation

279 (Berner,1980):

$280 \quad \frac{\partial Q}{\partial t}=\frac{\partial}{\partial z}\left(D b \frac{\partial Q}{\partial z}\right)+r(Q)$

281 where $\mathrm{Q}$ is the quantity of the tracer (e.g., luminophores), $\mathrm{t}$ is the time, $\mathrm{z}$ is the depth, $\mathrm{Db}$ is

282 the apparent biodiffusion coefficient, and $\mathrm{r}(\mathrm{Q})$ is the non-continuous displacement of tracer.

283 The term $r(Q)$ is defined as follows:

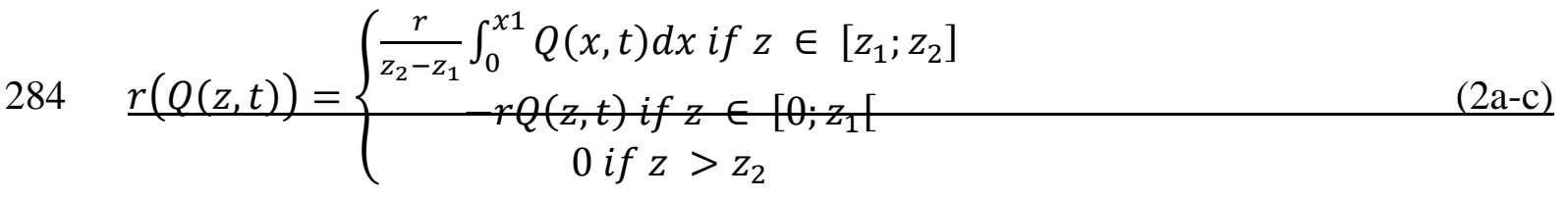


where $z_{1}$ and $z_{2}$ define the upper and lower limits of the tracer redistribution, $\mathrm{x}$ and $\mathrm{z}$ are depth variables and $r$ is the biotransport coefficient that is the percentage of tracer that left the $\left[\underline{0 ;} x_{1}\right]$ deposit and was redistributed in the $\left[\underline{z_{1} ; z_{2}}\right]$ layer. The redistribution of tracer between $z_{1}$ and $z_{2}$ and the disappearance of tracer from the $0-z_{1}$ layer are, respectively, described by Eqs. (2a) and (2b). Eq. (2c) indicates that no tracer movement occurs below $z_{2}$.

This displacement term was originally exemplified in a model describing gallerydiffusion of macrofaunal reworking (François et al., 2002). This biological reworking process describes the diffusive-like mixing of particles in the region of intense burrowing activity and the rapid transport of organic and inorganic material from the upper sediment layers to the lower regions of reworking (i.e. 'biotransport' or "non-local transport").

According to the experimental conditions, the following initial conditions were used:

$Q(z, O)=\left\{\begin{array}{c}Q_{0} \text { if } z \in\left[x_{1} ; x_{2}[\right. \\ \text { Oelse }\end{array}\right.$

297 where $\left[x_{1} ; x_{2}\right]$ is the tracer deposit layer. Finally, a zero-flux Neuman boundary condition was 298 considered:

$\frac{\partial Q}{\partial z}(0, t)=\lim _{z \rightarrow \infty} \frac{\partial Q}{\partial z}(z, t)=0$

300 The application of this bioturbation model to tracer redistributions, initially started by 301 François et al. (1997, 2001) and later revised by Duport al., (2007), allowed the quantification of two particle mixing coefficients: an apparent biodiffusion coefficient $\mathrm{Db}$ and a biotransport coefficient $\mathrm{r}$. The biodiffusion coefficient $\mathrm{Db}$ takes into account the diffusion-like transport

304 due to the activity of the organisms. We assume that the actual concentration dependent diffusion of tracers is negligible. The biotransport coefficient (r) represents a non-local mixing pattern associated with a biologically induced transfer of particles from one place to another in a discontinuous pattern (i.e. a non-continuous transport; Boudreau, 1986; Meysman et al., 308 2003). Estimates of the parameters Db and $r$ were finally obtained by minimizing a weighted 309 sum of squared differences between observed and calculated tracer concentrations (François 
et al., 1999, 2002). For each core, many adjustments between the observed and modelled

311 profiles are necessary in order to find the minimum weighted sum of squared differences.

This model was used with MatLab (@mathworks), thus it gives qualitative data (i.e.,

313 kind of sediment mixing) and quantitative data (intensity of the sediment mixing) on the

314 sediment mixing function for the entire benthic community at the sediment-water interface.

\subsection{Statistical analysis}

Bray-Curtis similarity matrix, based on square-root transformed data was used for the multivariate analysis of the macrobenthic community. Principal coordinate analysis (PCO) was conducted to explore multivariate variability among different sampling stations based on the $(\mathrm{B} / \mathrm{D})$ ratio community composition data matrix. Pearson rank correlation $(>0.5)$ vectors of species B/D with axes were overlaid on the PCO plots to visualize the relationships between ordination axes and the directions and degrees of variability in the biological variables. Differences in species composition in samples among the groups of stations were explored using non-parametric multivariate methods applied to Bray-Curtis dissimilarity matrix calculated from biomass/density ratio (B/D) (one-way PERMANOVA). Whenever the significant effect of factor was detected by the main PERMANOVA test, pair-wise tests for differences between levels of each significant factor was performed. SIMPER procedure (similarity percentage species contribution) was used to discriminate species responsible for

330 the differences between sites. In all models, a forward-selection procedure was used to 331 determine the best combination of predictor variables for explaining the variations in 332 macrofauna assemblages. The selection criteria chosen for the best-fitting relationship were 333 based on $\mathrm{R}^{2}$ values (Anderson et al. 2008). A distance-based linear model (DistLM) was used 334 to analyse and model the relationships between the macrofaunal community structure and the 
335 environmental factors. A distance-based redundancy analysis (dbRDA) was used to visualize

336 the variability along the two axes that best discriminated groups of samples defined by a priori

337 assigned groups. Superimposed vectors corresponded to Pearson's correlations (>0.5) of

338 environmental factors with the dbRDA axes. Calculations of the pseudo-F and p values were

339 based on 999 permutations of the residuals under a reduced model. The significance level for

340 all the statistical tests was $\mathrm{p}=0.05$.

341 The normality of environmental factors and biological factors (non-local and biodiffusion

342 coefficients, benthic density and biomass) was verified with use of Shapiro-Wilk test

343 ( $\mathrm{p}<0.05)$. Since data did not have a normal distribution, Spearman correlations were

344 calculated to estimate the relationships between faunal community characteristics (Table 8)

345 and environment (Appendix 1). Differences in benthic density, biomass, non-local and

346 biodiffusion coefficient were evaluated with the use of the nonparametric Kruskal-Wallis test,

347 and the Dunn's post-hoc multiple comparison test was applied to identify the differences

348 among stations groups. Station ST/8, sampled in July, was excluded from those analyses due

349 to lack of environmental information and because it was sampled during a different season

350 than the other stations. Additionally, a non-parametric pairwise Mann-Whitney U-test was

351 performed to compare differences between the spring and summer season in Storfjorden

352 (AX/3 vs ST/8). All analyses were performed using the PRIMER package v. 7 Clarke and

353 Gorley, 2006; Anderson et al., 2008) and the Statsoft software STATISTICA v. 9.

355 3. Results 
Bottom water salinity ranged from 34.5 to 35.1 and bottom water temperature ranged

360 from $-0.8{ }^{\circ} \mathrm{C}$ to $2.5{ }^{\circ} \mathrm{C}$ during our sampling. The lowest $\mathrm{BW} \mathrm{C}_{\text {org }}$ concentrations were 361 measured in Erik Eriksen Strait $(\mathrm{AX} / 4 ; 0.1 \pm 0.1 \%)$ and the highest in Storfjorden $(\mathrm{AX} / 3 ; 0.6$ $362 \pm 0.0 \%$ ). The BW $\delta^{13} \mathrm{C}$ values ranged from $-27.7 \%$ on the slope north of Svalbard (PS/32) to $363-22.2 \%$ in Storfjorden. The lowest BW C/N ratio values were found at the deepest station 364 (PS/31: $6.1 \pm 0.0)$ and the highest values were measured in the southern Barents Sea (AX/6: $36510.3 \pm 1.2$ ) (Table 2). Sandy and muddy sediments dominated in the study area. The lowest 366 SOM concentrations were measured at station PS/32, on slope $(2.6 \% \pm 0.1)$ and the highest in 367 Storfjorden $(\mathrm{AX} / 3 ; 6.5 \% \pm 0.3)$. The most depleted sediment $\delta^{13} \mathrm{C}$ values occurred in fjords 368 (AX/1: $-24.2 \%$ and $\mathrm{AX} / 2:-25.4 \%$ ) while the most enriched values were found on southern 369 Barents Sea shelf (AX/6: -22.2\%o). The lowest Sed C/N ratio values were found in deep basin 370 (PS/27: $7.8 \pm 0.4)$ and the highest value occurred in Van Mijenfjorden (AX/1: $18.7 \pm 0.5)$ 371 (Table 3). 
Table 2. Bottom water (BW) characteristics for each sampling station: $\mathrm{C}_{\mathrm{org}}, \mathrm{N}_{\mathrm{tot}}, \delta^{13} \mathrm{C}, \delta^{15} \mathrm{~N}$ (in $\%$ ) and $\mathrm{C} / \mathrm{N}$ values (mean $\pm \mathrm{SD}, \mathrm{n}=3$ ).

\begin{tabular}{|c|c|c|c|c|c|}
\hline Station & BW C $C_{\text {org }}(\%)$ & BW $\mathbf{N}_{\text {tot }}(\%)$ & $\mathrm{BW} \delta^{13} \mathrm{C}(\%)$ & $\mathrm{BW} \delta^{15} \mathrm{~N}(\%)$ & BW C/N \\
\hline $\mathbf{A X} / \mathbf{1}$ & $0.204 \pm 0.017$ & $0.023 \pm 0.002$ & $-24.7 \pm 0.1$ & $5.7 \pm 0.7$ & $10.3 \pm 1.1$ \\
\hline $\mathrm{AX} / 2$ & $0.187 \pm 0.005$ & $0.024 \pm 0.001$ & $-24.0 \pm 0.1$ & $3.9 \pm 0.5$ & $8.9 \pm 0.2$ \\
\hline $\mathbf{A X} / \mathbf{3}$ & $0.630 \pm 0.037$ & $0.097 \pm 0.005$ & $-22.2 \pm 0.2$ & $4.8 \pm 0.3$ & $7.6 \pm 0.2$ \\
\hline ST/8 & - & - & - & - & - \\
\hline $\mathrm{AX} / 4$ & $0.137 \pm 0.047$ & $0.015 \pm 0.003$ & $-24.0 \pm 0.1$ & $5.1 \pm 0.2$ & $10.2 \pm 1.7$ \\
\hline $\mathrm{AX} / 6$ & $0.268 \pm 0.013$ & $0.031 \pm 0.005$ & $-24.5 \pm 0.9$ & $5.9 \pm 1.7$ & $10.3 \pm 1.2$ \\
\hline PS/20 & $0.354 \pm 0.017$ & $0.063 \pm 0.001$ & $-23.5 \pm 0.0$ & $1.4 \pm 0.0$ & $6.6 \pm 0.2$ \\
\hline PS/32 & $0.180 \pm 0.002$ & $0.030 \pm 0.001$ & $-27.7 \pm 0.1$ & $1.9 \pm 0.6$ & $7.0 \pm 0.2$ \\
\hline PS/19 & $0.188 \pm 0.014$ & $0.032 \pm 0.001$ & $-24.6 \pm 0.2$ & $2.1 \pm 0.2$ & $6.8 \pm 0.2$ \\
\hline PS/27 & $0.226 \pm 0.007$ & $0.040 \pm 0.001$ & $-22.9 \pm 0.2$ & $2.0 \pm 0.7$ & $6.6 \pm 0.1$ \\
\hline PS/31 & $0.258 \pm 0.013$ & $0.050 \pm 0.002$ & $-23.8 \pm 0.1$ & $2.3 \pm 0.7$ & $6.1 \pm 0.0$ \\
\hline
\end{tabular}


381 Table 3. Sediment variables for each sampling station: sediment type, $\mathrm{C}_{\mathrm{org}}, \mathrm{N}_{\mathrm{tot}}, \delta^{13} \mathrm{C}, \delta^{15} \mathrm{~N}, \mathrm{OM}$ (in \%), C/N, Chl $a\left(\mu \mathrm{g}\right.$ DW g $\mathrm{g}^{-1}$ ) and Chl $a / \mathrm{Phaeo}$ 382 values (mean $\pm \mathrm{SD}, \mathrm{n}=$ no of cores).

\begin{tabular}{|c|c|c|c|c|c|c|c|c|c|c|c|c|c|}
\hline Station & $\begin{array}{c}\text { No } \\
\text { of } \\
\text { cores } \\
\end{array}$ & $\begin{array}{c}\text { Sediment } \\
\text { type }\end{array}$ & $\begin{array}{c}\text { Gravel } \\
(\%)\end{array}$ & $\begin{array}{c}\text { Sand } \\
(\%)\end{array}$ & $\underset{(\%)}{\text { Mud }}$ & $\begin{array}{c}\text { Sed C } C_{\text {org }} \\
(\%)\end{array}$ & $\begin{array}{c}\text { Sed } N_{\text {tot }} \\
(\%)\end{array}$ & $\begin{array}{c}\text { Sed } \\
\delta^{13} \mathrm{C} \\
(\% o) \\
\end{array}$ & $\begin{array}{c}\text { Sed } \\
\delta^{15} \mathbf{N} \\
(\% o) \\
\end{array}$ & Sed C/N & SOM (\%) & Chl $a(\mu \mathrm{g} / \mathrm{g})$ & Chl $a /$ Phaeo \\
\hline $\mathbf{A X} / \mathbf{1}$ & 3 & Sand & 0.0 & 78.1 & 21.9 & $1.9 \pm 0.0$ & $0.1 \pm 0.0$ & -24.2 & 4.0 & $18.7 \pm 0.5$ & $5.1 \pm 0.4$ & $2.3 \pm 0.2$ & $0.5 \pm 0.1$ \\
\hline $\mathrm{AX} / 2$ & 3 & Sand & 1.3 & 86.8 & 12.0 & $1.8 \pm 0.0$ & $0.1 \pm 0.0$ & -25.4 & 4.8 & $18.1 \pm 0.2$ & $4.4 \pm 0.3$ & $1.4 \pm 0.1$ & $0.4 \pm 0.0$ \\
\hline $\mathbf{A X} / \mathbf{3}$ & 3 & Mud & 0.0 & 40.8 & 59.2 & $2.1 \pm 0.0$ & $0.2 \pm 0.0$ & -22.9 & 3.6 & $10.8 \pm 0.1$ & $6.5 \pm 0.3$ & $19.4 \pm 2.6$ & $1.9 \pm 0.4$ \\
\hline ST/8 & 2 & - & - & - & - & $2.1 \pm 0.0$ & $0.2 \pm 0.0$ & - & & $11.0 \pm 0.4$ & $7.3 \pm 0.6$ & $21.8 \pm 1.3$ & $51.8 \pm 34.2$ \\
\hline $\mathrm{AX} / 4$ & 3 & Sand & 2.3 & 66.4 & 31.2 & $1.0 \pm 0.0$ & $0.1 \pm 0.0$ & -23.6 & 5.3 & $8.2 \pm 0.2$ & $5.1 \pm 0.6$ & $2.2 \pm 0.3$ & $0.3 \pm 0.0$ \\
\hline $\mathrm{AX} / 6$ & 3 & Mud & 1.3 & 42.6 & 56.1 & $2.1 \pm 0.0$ & $0.3 \pm 0.0$ & -22.2 & 4.6 & $9.9 \pm 0.2$ & $5.7 \pm 0.6$ & $2.6 \pm 0.5$ & $0.4 \pm 0.0$ \\
\hline PS/20 & 2 & Sand & 7.5 & 51.3 & 41.2 & $0.9 \pm 0.0$ & $0.1 \pm 0.0$ & -22.8 & 2.8 & $8.5 \pm 0.1$ & $4.6 \pm 0.3$ & $7.6 \pm 1.7$ & $1.1 \pm 0.2$ \\
\hline PS/32 & 2 & Sand & 1.9 & 76.6 & 21.5 & $0.5 \pm 0.1$ & $0.1 \pm 0.0$ & -22.8 & 4.0 & $8.1 \pm 0.2$ & $2.6 \pm 0.1$ & $12.8 \pm 1.6$ & $1.9 \pm 0.4$ \\
\hline PS/19 & 3 & Sand & 0.1 & 74.5 & 25.3 & $1.6 \pm 0.0$ & $0.2 \pm 0.0$ & -22.7 & 4.6 & $9.5 \pm 0.3$ & $8.3 \pm 0.8$ & $2.9 \pm 0.5$ & $0.4 \pm 0.0$ \\
\hline PS/27 & 3 & Sand & 6.3 & 62.3 & 31.5 & $0.8 \pm 0.0$ & $0.1 \pm 0.0$ & -22.9 & 3.0 & $7.8 \pm 0.4$ & $3.7 \pm 0.3$ & $2.6 \pm 0.5$ & $0.5 \pm 0.0$ \\
\hline PS/31 & 3 & Sand & 30.1 & 46.0 & 23.9 & $0.8 \pm 0.1$ & $0.1 \pm 0.0$ & -23.0 & 3.9 & $16.7 \pm 3.8$ & $4.4 \pm 0.1$ & $1.1 \pm 0.4$ & $0.3 \pm 0.0$ \\
\hline
\end{tabular}

383 
to 68 (PS/32) (Table 4). Four burrowing and four sediment-mixing types were recorded. Subin Svalbard fjords in biomass and density, and in Storfjorden in density. The deep burrowing Yoldia hyperborea (conveyor) dominated in biomass at AX/3. Two biodiffusors, the tube building polychaete Myriochele heeri and the deep burrowing bivalve Astarte borealis dominated in Erik Eriksen station (AX/4) in density and biomass respectively. The tube building Spiochaetopterus typicus (conveyor) dominated in terms of density and was second dominant in biomass in the Southern Barents Sea (AX/6). The sea star Ctenodiscus sp. 400 dominated in biomass at this station. The tube building polychaete, Maldane glebifex, dominated in both density and biomass at the shelf station PS/20. Deep burrowing bivalves (Yoldiella lenticula, Yoldia hyperborea) dominated in density at PS/32 while the tube building polychaete Galathowenia oculata dominated in biomass. Burrow-building taxa were mostly biodiffusors and dominated at all shallow stations. Deep burrowing and tube building taxa were mostly conveyor bioturbators and dominated at deeper stations (Table 4). Fourteen mobility-feeding groups were recorded, and sessile and mobile macrofauna dominated at all stations except from the deepest one (PS/31) where discretely mobile fauna dominated. The 408 lowest number of functional groups was found in Hornsund (AX/2) where 4 groups (sessile 409 surface feeders, discretely subsurface feeders, mobile omnivore and mobile subsurface 410 feeders) occurred. Sessile subsurface feeders dominated at PS/20 (30\%) and PS/27 (33\%). 411 Sessile surface feeders were predominant in fjords (AX/1: 52\%; AX/2: 80\%), Storfjorden $412(\mathrm{AX} / 3: 35 \%)$, in the southern Barents Sea (AX/6: 45\%) and on slope (PS/19: 18\%). The share 413 of discretely mobile fauna increased with depth, and discretely mobile surface feeders 
414 dominated in the Nansen Basin (PS/31: 44\%). The highest number of mobile subsurface

415 feeders was found on the shelf (PS/32: 23\%). The number of mobile taxa was similar for all

416 stations. The mobile surface fauna dominated in Erik Eriksen Strait (AX/4: 25\%) (Fig. 2).

417

418

419

420

421

422

423

424

425

426

427

428

429

430

431

432

433

434

435

436

437

438

439

440 
Table 4. Functional traits, relative density and biomass of the three dominant taxa for each sampling station. Class: P - Polychaeta, B - Bivalvia,

442 An - Anthozoa, As - Asteroidea, O - Ophiuroidea, S - Sipunculidea. Mobility and feeding groups (M/F) are marked by codes: mobility type (D -

Discretely mobile, M - Mobile, S - Sessile) and feeding type (car - carnivore, omn - omnivore, sub - subsurface feeder, sur - surface feeder, sus suspension feeder). Burrowing depth (BT): 1 - surface burrowing, 2 - subsurface burrowing, 3 - deep burrowing. Tubes (T): “+” - I-shaped tube, “_“- - no tube. Sediment mixing types (SMix): biodiffusor (B), upward conveyor (UC), gallery diffusor (GD), downward conveyor (DC).

\begin{tabular}{|c|c|c|c|c|c|c|c|c|c|c|c|c|c|c|c|}
\hline Station & $\begin{array}{c}\text { No } \\
\text { of } \\
\text { taxa }\end{array}$ & Taxa & Class & $\mathbf{M} / \mathbf{F}$ & BT & $\mathbf{T}$ & SMix & $\begin{array}{c}\text { Density } \\
\%\end{array}$ & Taxa & Class & $\mathbf{M} / \mathbf{F}$ & BT & $\mathbf{T}$ & SMix & $\begin{array}{c}\text { Biomass } \\
\%\end{array}$ \\
\hline \multirow[t]{3}{*}{$\mathbf{A X} / \mathbf{1}$} & 20 & Cirratulidae $^{2}$ & $\mathrm{P}$ & Ssur & 2 & - & B & 41.4 & Lumbrineris sp. ${ }^{6}$ & $\mathrm{P}$ & Momn & 2 & - & GD & 72.1 \\
\hline & & Polycirrus arcticus ${ }^{4,5}$ & $\mathrm{P}$ & Ssur & 3 & + & DC & 7.1 & Polycirrus arcticus ${ }^{4,5}$ & $\mathrm{P}$ & Ssur & 3 & + & $\mathrm{DC}$ & 11.5 \\
\hline & & Lumbrineris sp. ${ }^{6}$ & $\mathrm{P}$ & Momn & 2 & - & GD & 6.4 & Aglaophamus malmgreni ${ }^{4}$ & $\mathrm{P}$ & Mcar & 2 & - & B & 10.6 \\
\hline \multirow[t]{3}{*}{$\mathrm{AX} / 2$} & 9 & Cirratulidae $^{2}$ & $\mathrm{P}$ & Ssur & 2 & - & B & 66.7 & Cirratulidae $^{2}$ & $\mathrm{P}$ & Ssur & 2 & - & B & 49.3 \\
\hline & & Polycirrus arcticus ${ }^{4,5}$ & $\mathrm{P}$ & Ssur & 3 & + & DC & 13.1 & Polycirrus arcticus ${ }^{4,5}$ & $\mathrm{P}$ & Ssur & 3 & + & $\mathrm{DC}$ & 36.6 \\
\hline & & Lumbrineris sp. ${ }^{6}$ & $\mathrm{P}$ & Momn & 2 & & GD & 8.3 & Lumbrineris sp. ${ }^{6}$ & $\mathrm{P}$ & Momn & 2 & - & GD & 8.7 \\
\hline \multirow[t]{3}{*}{$\mathbf{A X} / \mathbf{3}$} & 34 & Cirratulidae $^{2}$ & $\mathrm{P}$ & Ssur & 2 & & B & 31.2 & Yoldia hyperborea $^{7}$ & B & Msub & 3 & - & $\mathrm{C}$ & 57.4 \\
\hline & & Lumbrineris sp. ${ }^{6}$ & $\mathrm{P}$ & Momn & & & GD & 14.1 & Maldane sarsi ${ }^{8}$ & $\mathrm{P}$ & Ssub & 3 & + & $\mathrm{C}$ & 12 \\
\hline & & Yoldia hyperborea $^{7}$ & $\mathrm{~B}$ & Msub & & - & $\mathrm{C}$ & 6.3 & Nuculana radiata ${ }^{4}$ & B & Msub & 3 & - & $\mathrm{B}$ & 11.5 \\
\hline \multirow[t]{3}{*}{ ST/8 } & 29 & Lumbrineris sp. ${ }^{6}$ & $\mathrm{P}$ & Momn & 2 & - & GD & 18.3 & Yoldia hyperborea $^{7}$ & B & Msub & 3 & - & $\mathrm{C}$ & 30.5 \\
\hline & & Cirratulidae $^{2}$ & $\mathrm{P}$ & Ssur & 2 & - & B & 11 & Nuculana radiata ${ }^{4}$ & B & Msub & 3 & - & B & 27.8 \\
\hline & & Eteone longa ${ }^{9,10}$ & $\mathrm{P}$ & Msub & 1 & - & GD & 7.3 & Macoma calcarea $^{11}$ & B & Ssur & 3 & - & B & 13.7 \\
\hline $\mathrm{AX} / 4$ & 63 & Myriochele heeri ${ }^{17}$ & $\mathrm{P}$ & Msur & 3 & + & $\mathrm{B}$ & 12.1 & Astarte borealis ${ }^{4}$ & $\mathrm{~B}$ & Msus & 3 & - & $\mathrm{B}$ & 90 \\
\hline
\end{tabular}




\begin{tabular}{|c|c|c|c|c|c|c|c|c|c|c|c|c|c|c|c|}
\hline & & Macoma sp. ${ }^{1,11}$ & B & Ssur & 3 & - & B & 11.6 & Actinaria $^{4}$ & An & Scar & 1 & - & B & 1.8 \\
\hline & & Maldane sarsi ${ }^{8}$ & $\mathrm{P}$ & Ssub & 3 & + & $\mathrm{C}$ & 8.2 & Yoldiella lenticula ${ }^{7}$ & B & Msur & 3 & - & $\mathrm{C}$ & 1.5 \\
\hline \multirow[t]{3}{*}{$\mathrm{AX} / 6$} & 36 & Spiochaetopterus typicus $^{8}$ & $\mathrm{P}$ & Ssur & 3 & + & $\mathrm{C}$ & 34.9 & Ctenodiscus sp. ${ }^{20}$ & As & Msur & 1 & - & $\mathrm{B}$ & 47.3 \\
\hline & & Macoma sp. ${ }^{1,11}$ & B & Ssur & 3 & - & $\mathrm{B}$ & 6.4 & Spiochaetopterus typicus $^{8}$ & $\mathrm{P}$ & Ssur & 3 & + & $\mathrm{C}$ & 27.3 \\
\hline & & Heteromastus sp. ${ }^{12,13,14}$ & $\mathrm{P}$ & Msub & 3 & - & $\mathrm{C}$ & 6.4 & Aglaophamus malmgreni ${ }^{4}$ & $\mathrm{P}$ & Mcar & 2 & - & $\mathrm{B}$ & 6.4 \\
\hline \multirow[t]{3}{*}{ PS/20 } & 58 & Maldane glebifex ${ }^{8}$ & $\mathrm{P}$ & Ssub & 3 & + & $\mathrm{C}$ & 22.4 & Maldane glebifex ${ }^{8}$ & $\mathrm{P}$ & Ssub & 3 & + & $\mathrm{C}$ & 24.5 \\
\hline & & Yoldiella lenticula $^{7}$ & B & Msur & 3 & - & $\mathrm{C}$ & 8.7 & Chirimia biceps $^{8}$ & $\mathrm{P}$ & Ssub & 3 & + & $\mathrm{C}$ & 9.4 \\
\hline & & Macoma calcarea ${ }^{11}$ & B & Ssur & 3 & - & B & 7.1 & Nicomache lumbricalis ${ }^{8}$ & $\mathrm{P}$ & Ssub & 3 & + & $\mathrm{C}$ & 9.4 \\
\hline \multirow[t]{3}{*}{ PS/32 } & 68 & Yoldiella lenticula $^{7}$ & $\mathrm{~B}$ & Msur & 3 & - & $\mathrm{C}$ & 13.8 & Galathowenia oculata $^{3}$ & $\mathrm{P}$ & Msur & 2 & + & $\mathrm{C}$ & 7.7 \\
\hline & & Yoldia hyperborea ${ }^{7}$ & B & Msub & 3 & - & $\mathrm{C}$ & 8.7 & Ctenodiscus sp. ${ }^{20}$ & As & Msur & 1 & - & $\mathrm{B}$ & 7.5 \\
\hline & & Axinopsida orbiculata ${ }^{15}$ & B & Dsub & 3 & - & $\mathrm{C}$ & 5.9 & Yoldiella lenticula $^{7}$ & B & Msur & 3 & - & $\mathrm{C}$ & 6.3 \\
\hline \multirow[t]{3}{*}{ PS/19 } & 38 & Cirratulidae $^{2}$ & $\mathrm{P}$ & Ssur & 2 & - & $\mathrm{B}$ & 12 & Amphiura sundevalli ${ }^{4}$ & $\mathrm{O}$ & Msus & 1 & - & $\mathrm{B}$ & 25.5 \\
\hline & & Notoproctus oculatus ${ }^{8}$ & $\mathrm{P}$ & Ssub & 3 & & C & 10.1 & Lumbrineridae $^{6}$ & $\mathrm{P}$ & Somn & 2 & - & GD & 9.3 \\
\hline & & Yoldia hyperborea $^{7}$ & $\mathrm{~B}$ & Msub & 3 & & C & 8.9 & Nemertea $^{4}$ & $\mathrm{~N}$ & Momn & 1 & - & $\mathrm{B}$ & 7.1 \\
\hline \multirow[t]{3}{*}{ PS/27 } & 35 & Prionospio cirrifera $^{16}$ & $\mathrm{P}$ & Dsur & 2 & & $\mathrm{C}$ & 13.2 & Streblosoma intestinale 4 & $P$ & Dsur & 3 & + & $\mathrm{C}$ & 43 \\
\hline & & Notoproctus oculatus $^{8}$ & $\mathrm{P}$ & Ssub & 3 & + & $\mathrm{C}$ & 13.2 & Chone fauveli $^{3}$ & $P$ & Ssur & 2 & + & $\mathrm{C}$ & 32.6 \\
\hline & & Lumbriclymene minor ${ }^{8}$ & $P$ & Ssub & 3 & + & $\mathrm{C}$ & 8.8 & Notoproctus oculatus ${ }^{8}$ & $\mathrm{P}$ & Ssub & 3 & + & $\mathrm{C}$ & 4.2 \\
\hline \multirow[t]{3}{*}{ PS/31 } & 19 & Levinsenia gracilis ${ }^{18}$ & $P$ & Dsur & 2 & - & $\mathrm{C}$ & 33.6 & Nephasoma lilljeborgi ${ }^{19}$ & $S$ & Dsur & 3 & - & $\mathrm{C}$ & 28 \\
\hline & & Paraonidae $^{2}$ & $P$ & Msub & 2 & - & B & 19.3 & Levinsenia gracilis ${ }^{18}$ & $\mathrm{P}$ & Dsur & 2 & - & $\mathrm{C}$ & 14.2 \\
\hline & & Cirrophorus sp. $^{2}$ & $\mathrm{P}$ & Dsub & 2 & - & B & 16.8 & Paraonidae $^{2}$ & $\mathrm{P}$ & Msub & 2 & - & B & 11 \\
\hline
\end{tabular}


447 References in superscripts: ${ }^{1}$ Gilbert at al. (2007); ${ }^{2}$ Gérino at al. (1992, 2007); ${ }^{3}$ Fauchald and Jumars (1979); ${ }^{4}$ Queirós at al. (2013); ${ }^{5}$ Gingras et 448 al. (2008); ${ }^{6}$ Petch (1986); ${ }^{7}$ Stead and Thompson (2006); ${ }^{8}$ Smith and Shafer (1984); ${ }^{9}$ Mazik and Elliott (2000); ${ }^{10}$ Mermillod-Blondin et al. 449 (2003); ${ }^{11}$ Michaud et al. (2006); ${ }^{12}$ D'Andrea et al. (2004); ${ }^{13}$ Mulsow et al. (2002); ${ }^{14}$ Quintana et al. (2007); ${ }^{15}$ Zanzerl and Dufour (2017); ${ }^{16}$ 450 Bouchet et al. (2009); ${ }^{17}$ Duchêne and Rosenberg (2001); ${ }^{18}$ Venturini et al. (2011); ${ }^{19}$ Shields and Kędra (2009); ${ }^{20}$ Shick (1976). 


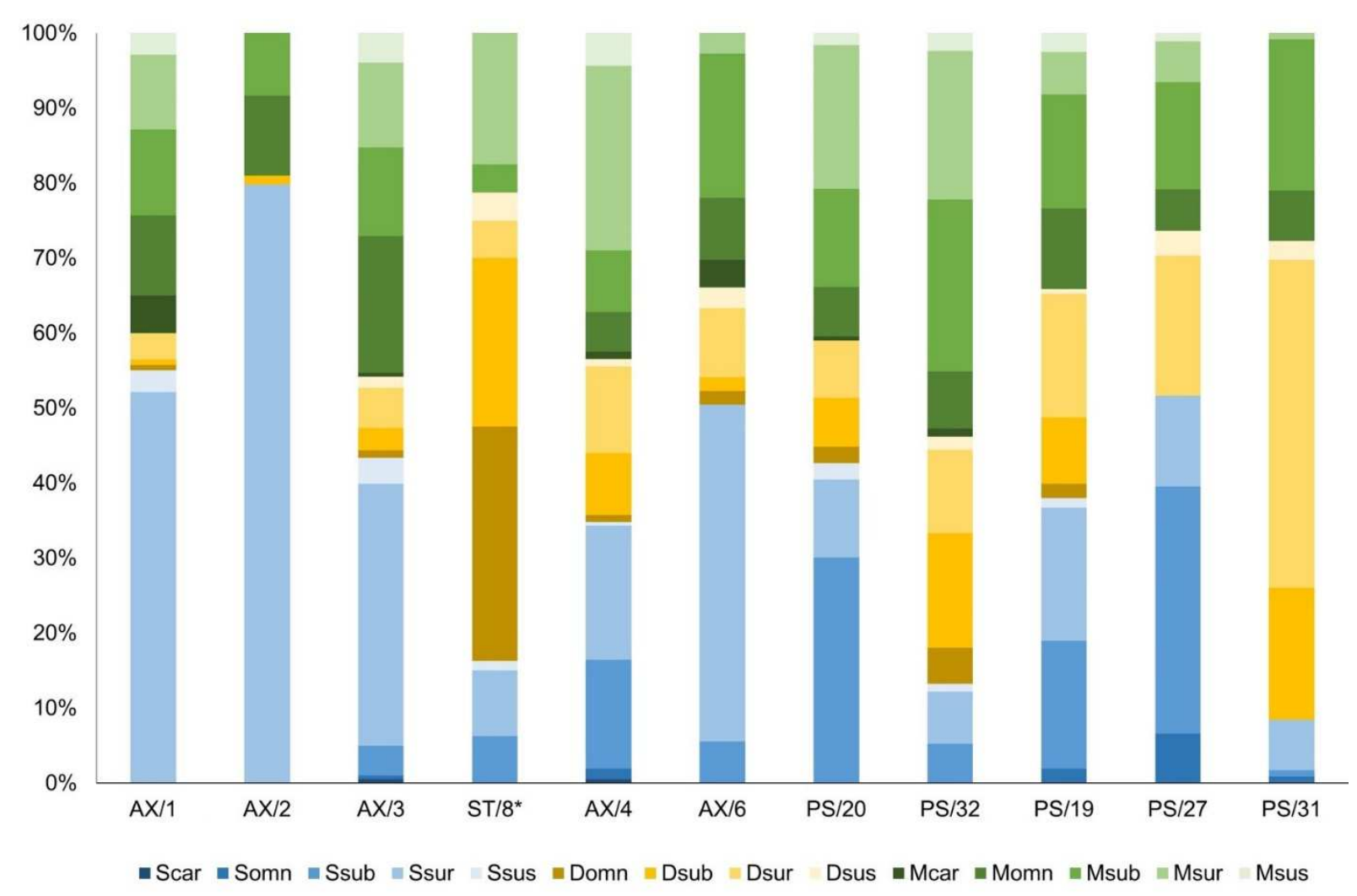

453 Fig. 2. Percentages of mobility and feeding groups at different sampling stations. Station ST/8 454 marked with * was sampled in summer season. Functional traits codes: mobility type (D 455 Discretely mobile (yellow), M - Mobile (green), S - Sessile (blue)) and feeding type (car 456 carnivore, omn - omnivore, sub - subsurface feeder, sur - surface feeder, sus - suspension 457 feeder).

458 Stations were separated into 4 groups, based on the PCO analysis: A - fjords (Van 459 Mijenfjorden: AX/1, Hornsund: AX/2), B - Storfjorden (AX/3), C - Barents Sea shelf (Erik 460 Eriksen Strait: AX/4, southern Barents Sea: AX/6, and northern Barents Sea: PS/20, PS/32), D 461 - northern Barents Sea, stations deeper than 400m on continental stock: PS/19, PS/27 and 462 Nansen Basin: PS/31. PCO explained $22.5 \%$ of the variability among sampling stations: the 463 first axis explained $13.6 \%$ and the second axis $8.9 \%$ (Fig. 3). Fjords' communities were 464 correlated with presence of polychaete Polycirrus arcticus and cumacean Diastylis lucifera while benthic patterns in Storfjorden were correlated with presence of polychaetes Maldane 
466 sarsi and Apistobranchus tullbergi, and bivalves Musculus discors, Ennucula tenuis and

467 Yoldia hyperborea. Those correlations were negative for deeper stations where benthic 468 communities were mainly correlated with presence of polychaetes Notoproctus oculatus and 469 Prionospio cirrifera. The shelf stations varied the most with less clear patterns for benthic 470 communities.

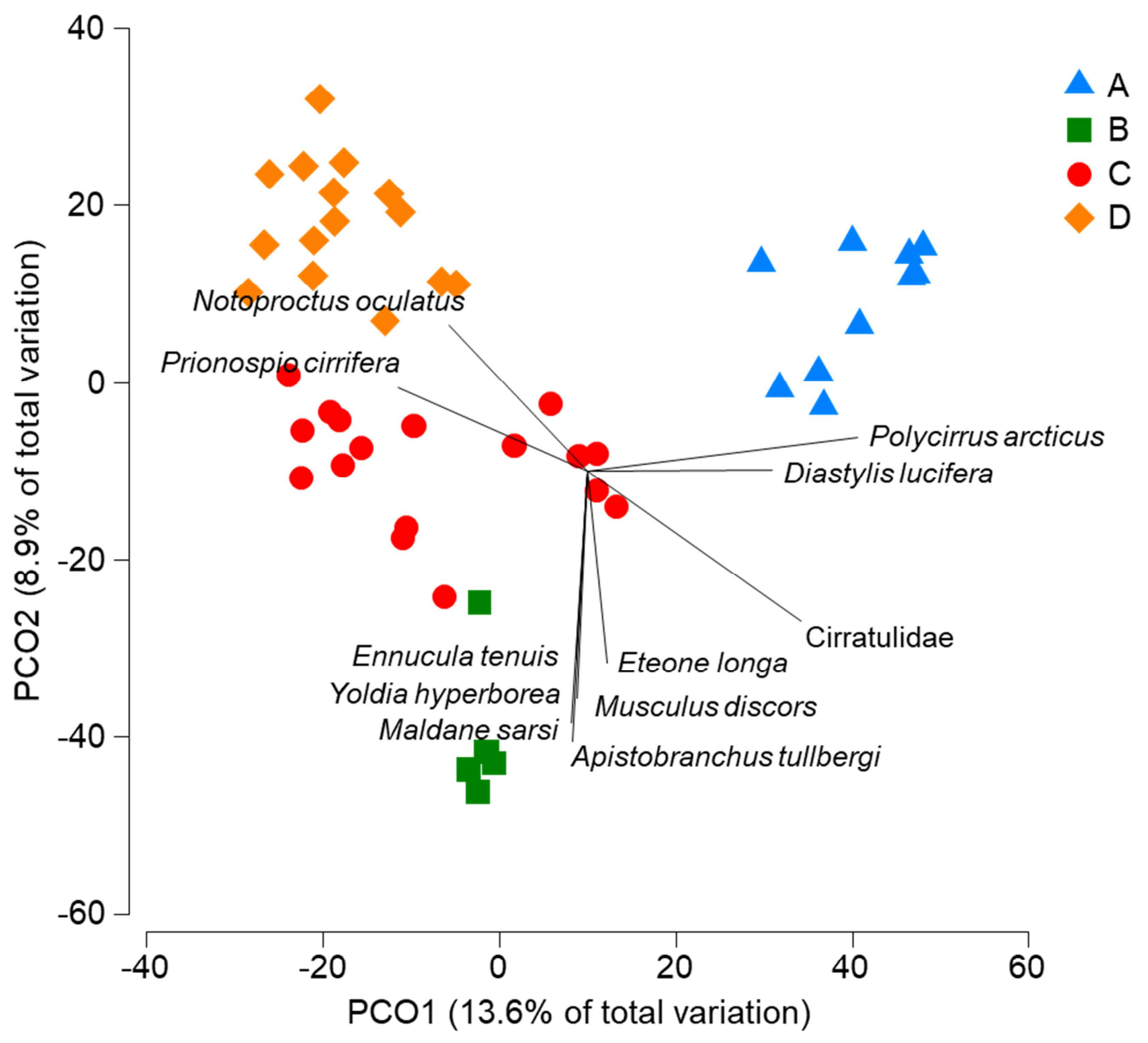

472 Fig. 3. PCO analysis for macrobenthic communities based on species biomass to density ratio,

473 and the Bray-Curtis similarity among four sampling areas: A (Hornsund, Van Mijenfjorden);

474 B (Storfjorden); C (Barents Sea shelf); D (northern Barents Sea and Nansen Basin).

475 Significantly correlated species with the PCO coordinates $(r>0.5)$ are shown on the plot. 
Polychaeta dominated at all stations. There were significant differences in density

478 (Kruskal-Wallis test; $\mathrm{p}<0.05$; significant differences (post hoc test) between group A:B, A:C 479 and C:D) (Fig. 4A). Benthic density ranged from 1485.5 ind. $/ \mathrm{m}^{2} \pm 168.7$ standard error (SE) 480 (station $\mathrm{AX} / 2$ ) to 2475.8 ind. $/ \mathrm{m}^{2} \pm 369.9 \mathrm{SE}$ (station $\mathrm{AX} / 1$ ) in group A. In group B benthic 481 density was 3625.3 ind./ $/ \mathrm{m}^{2} \pm$ 83.9 SE (station AX/3). In group C density ranged from 1927.6 482 ind. $/ \mathrm{m}^{2} \pm 196.5 \mathrm{SE}$ (station $\mathrm{AX} / 6$ ) to $6388.5 \mathrm{ind} . / \mathrm{m}^{2} \pm 399.3 \mathrm{SE}$ (station PS/32). In group D 483 density ranged from 1609.3 ind. $/ \mathrm{m}^{2} \pm 295.1 \mathrm{SE}$ (station PS/27) to 2794.1 ind. $/ \mathrm{m}^{2} \pm 404.8 \mathrm{SE}$ 484 (station PS/19). There were significant differences in biomass among areas (Kruskal-Wallis 485 test; $\mathrm{p}<0.05$; significant differences (post hoc test) between group A:B, B:D and C:D) (Fig. 486 4B). Benthic biomass ranged from $2.6 \mathrm{~g}$ wet weight $/ \mathrm{m}^{2} \pm 0.5 \mathrm{SE}$ (station $\mathrm{AX} / 2$ ) to $37 \mathrm{~g}$ wet 487 weight $/ \mathrm{m}^{2} \pm 18.4 \mathrm{SE}$ (station $\mathrm{AX} / 1$ ) in group A. In group B benthic biomass was $172.3 \mathrm{~g}$ wet 488 weight $/ \mathrm{m}^{2} \pm 37.3 \mathrm{SE}$ (station $\mathrm{AX} / 3$ ). In group $\mathrm{C}$ biomass ranged from $7.8 \mathrm{~g}$ wet weight $/ \mathrm{m}^{2} \pm$ 489 1.1 SE (station PS/32) to $112.9 \mathrm{~g}$ wet weight $/ \mathrm{m}^{2} \pm 61.3 \mathrm{SE}$ (station AX/4). In group D biomass 490 ranged from $2.2 \mathrm{~g}$ wet weight $/ \mathrm{m}^{2} \pm 0.5 \mathrm{SE}$ (station PS/31) to $11.2 \mathrm{~g}$ wet weight $/ \mathrm{m}^{2} \pm 5.7 \mathrm{SE}$ 491 (station PS/27).

492

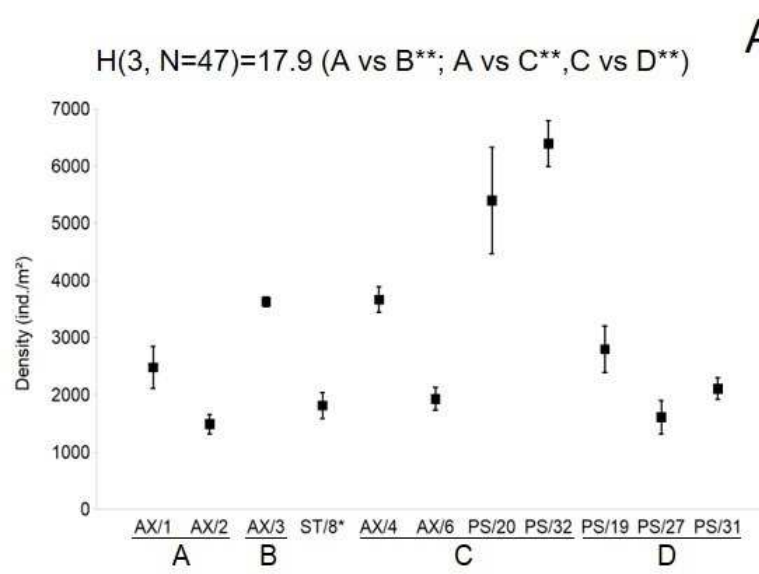

A

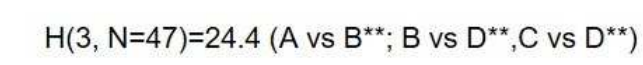

B

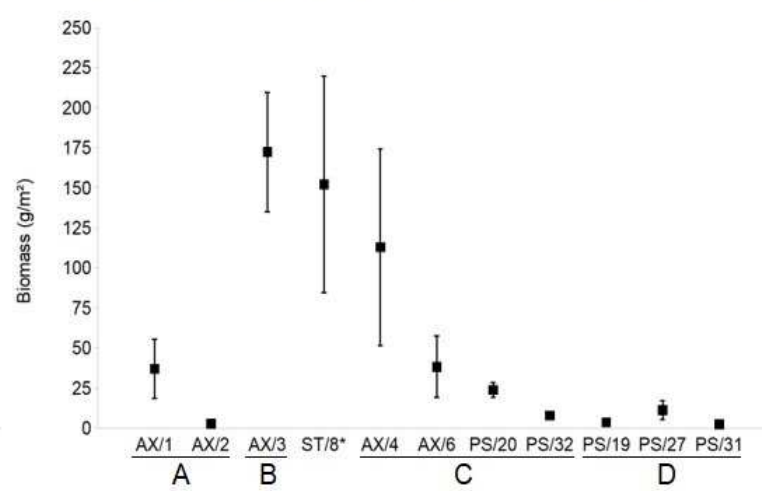

493 Fig. 4. Mean density (ind. $\left./ \mathrm{m}^{-2}\right)(\mathrm{A})$ and biomass $\left(\mathrm{g} / \mathrm{m}^{-2}\right)(\mathrm{B}) ; \pm \mathrm{SE}, \mathrm{n}=$ no of cores (Table 1$)$ at 494 stations sampled in Van Mijenfjorden, Hornsund (group A); Storfjorden (group B); Barents 495 Sea shelf (group C); northern Barents Sea and Nansen Basin (group D). Station ST/8 marked 
496 with * was sampled in summer season. Kruskal - Wallis results for differences between

497 sampling sites are given; significant test results are marked with $* *(\mathrm{p}<0.05)$.

498 There were significant differences in the benthic communities structure

499 (biomass/density ratio) among different locations (PERMANOVA test Pseudo-F : 5.07, $500 \mathrm{p}=0.001$ ). Significant differences were found for each group (significant pairwise 501 comparisons $\mathrm{p}=0.001$ ); see Table 5 for details.

502

503 Table 5. PERMANOVA results for the multivariate descriptors of benthic communities with 504 significant pair-wise comparisons results for different groups.

\begin{tabular}{llcccc}
\hline Benthic parameter & Source of variation & Df & MS & Pseudo-F & P (perm) \\
\hline Biomass/Density ratio & Gr & 3 & 16606.0 & 5.07 & 0.001 \\
& Res & 43 & 3272.8 & & \\
& Total & 46 & & &
\end{tabular}

\begin{tabular}{lllcccc}
\hline Benthic parameter & Regime & Site & t & Df & P(MC) & P (perm) \\
\hline Biomass/Density ratio & Groups & A:B & 2.886 & 13 & 0.001 & 0.001 \\
& & A:C & 2.469 & 25 & 0.001 & 0.001 \\
& & A:D & 2.715 & 23 & 0.001 & 0.001 \\
& & B:C & 1.874 & 20 & 0.001 & 0.001 \\
& & B:D & 2.151 & 18 & 0.001 & 0.001 \\
& & C:D & 1.852 & 30 & 0.001 & 0.001 \\
\hline
\end{tabular}

505

506

Benthic taxa that contributed mostly to the groups similarities were: Polycirrus arcticus (44.7\%) in fjords (A), Yoldia hyperborea (31.7 \%) in Storfjorden (B),

509 Spiochaetopterus typicus $(16.8 \%)$ in the Barents Sea shelf (C) and Nephasoma diaphanes

510 diaphanes $(16 \%)$ in the northern Barents Sea and Nansen Basin (D) as revealed by SIMPER 511 analysis (Table 6).

513 Table 6. SIMPER analysis results based on B/D ratio. Species that contributed more than 5\% 514 of the average similarity for different sampling stations groups are listed. 
ACCEPTED MANUSCRIPT

\begin{tabular}{cclc}
\hline Group & Average similarity & Species & Contribution \% \\
\hline A & 39.7 & Polycirrus arcticus & 44.7 \\
& & Cirratulidae & 28.5 \\
& & Lumbrineris sp. & 18.7 \\
B & 35.9 & Yoldia hyperborea & 31.7 \\
& & Maldane sarsi & 31 \\
& & Nuculana radiata & 9 \\
& & Lumbrineris sp. & 8.1 \\
& & Cirratulidae & 5.8 \\
C & \multirow{2}{*}{12} & Spiochaetopterus typicus & 16.8 \\
& & Lumbrineris sp. & 10.7 \\
& & Yoldiella lenticula & 7.3 \\
& & Maldane sarsi & 5.6 \\
D & & Nephasoma diaphanes diaphanes & 16 \\
& & Maldane glebifex & 11.7 \\
& & Prionospio cirrifera & 10.1 \\
& & Notoproctus oculatus & 9.8 \\
& & Nemertea & 9.5 \\
& & Lumbrineris sp. & 6.3 \\
& & Byblis minuticornis & 5.9 \\
\hline
\end{tabular}
observed in the macrofauna community while Sed $\delta^{13} \mathrm{C}(10 \%)$ and Sed C/N (9.3\%) were next 518 main contributors. Nine variables were included by the DistLM procedure to construct the 519 best fitting model, together explaining $46.8 \%$ of total variation. However, one of the variables was not statistically significant (gravel) (Table 7). The most important parameter contributing to the first axis of the dbRDA plot was Sed $\mathrm{C} / \mathrm{N}$ and explained $17.2 \%$ of fitted variation. It also positively correlated with fjords' group (A). The most important parameter contributing 523 to the second axis was sediment $\mathrm{Chl} a$ and explained $25.7 \%$ of fitted flux variation. It was 524 positively correlated with Storfjords group (B) and most stations in group C (shelf) (Fig. 5).

Table 7. Results of DistLM procedure for fitting environmental variables to the macofauna community data. \%Var - percentage of explained variance; \%Cum - cumulative percentage 528 explained by the added variable. Significance level $\mathrm{p}<0.05$. Environmental factors: D 529 depth, S - salinity, T - temperature, types of sediment (mud, sand, gravel), BW $\mathrm{C}_{\text {org }}$ - bottom 530 water $\mathrm{C}_{\text {org }}$, BW $\mathrm{N}_{\text {tot }}-$ bottom water $\mathrm{N}_{\text {tot }}$, BW $\delta^{13} \mathrm{C}-$ bottom water $\delta^{13} \mathrm{C} \mathrm{BW}, \mathrm{BW} \delta^{15} \mathrm{~N}-$ 
531 bottom water $\delta^{15} \mathrm{~N}, \mathrm{BW} \mathrm{C} / \mathrm{N}-$ bottom water $\mathrm{C} / \mathrm{N}$, Sed $\mathrm{C}_{\mathrm{org}}-\mathrm{C}_{\mathrm{org}}$ concentration in sediment,

532 Sed $\mathrm{N}_{\text {tot }}-$ sediment $\mathrm{N}_{\text {tot }}$, Sed $\delta^{13} \mathrm{C}-$ sediment $\delta^{13} \mathrm{C}$, Sed $\delta^{15} \mathrm{~N}-$ sediment $\delta^{15} \mathrm{~N}$, Sed $\mathrm{C} / \mathrm{N}-$

533 sediment $\mathrm{C} / \mathrm{N}, \mathrm{SOM}$ - sediment organic matter, Chl $a$ - sediment Chlorophyll $a$ and Chl

$534 a /$ Phaeo - sediment Phaeopigments.

\begin{tabular}{|c|c|c|c|c|c|}
\hline \multicolumn{6}{|c|}{ MARGINAL TESTS } \\
\hline Variable & Pseudo-F & Var\% & $\mathrm{P}$ & $\infty$ & 7 \\
\hline $\mathrm{S}$ & 5.06 & 10.1 & 0.001 & & \\
\hline Sed $\delta^{13} \mathrm{C}$ & 5.01 & 10.0 & 0.001 & & \\
\hline Sed $\mathrm{C} / \mathrm{N}$ & 4.59 & 9.3 & 0.001 & & \\
\hline $\mathrm{BW} \mathrm{C} / \mathrm{N}$ & 4.31 & 8.7 & 0.001 & & \\
\hline $\mathrm{BW} \delta^{15} \mathrm{~N}$ & 4.25 & 8.6 & 0.001 & & \\
\hline Sed $\mathrm{C}_{\text {org }}$ & 4.11 & 8.4 & 0.001 & & \\
\hline $\mathrm{D}$ & 3.99 & 8.1 & 0.001 & & \\
\hline $\mathrm{T}$ & 3.96 & 8.1 & 0.001 & & \\
\hline Chl $a$ & 3.66 & 7.5 & 0.001 & & \\
\hline Sand & 3.62 & 7.4 & 0.001 & & \\
\hline Mud & 3.46 & 7.1 & 0.001 & & \\
\hline Chl $a$ / Phaeo & 3.34 & 6.9 & 0.001 & & \\
\hline $\mathrm{BW} \mathrm{C}_{\text {org }}$ & 3.26 & 6.8 & 0.001 & & \\
\hline $\mathrm{BW} \mathrm{N}_{\text {tot }}$ & 3.16 & 6.6 & 0.002 & & \\
\hline Gravel & 3.11 & 6.5 & 0.001 & & \\
\hline Sed $\mathrm{N}_{\text {tot }}$ & 2.43 & 5.1 & 0.001 & & \\
\hline $\operatorname{Sed} \delta^{15} N$ & 2.09 & 4.4 & 0.004 & & \\
\hline $\mathrm{BW} \delta^{13} \mathrm{C}$ & 2.04 & 4.3 & 0.001 & & \\
\hline SOM & 1.56 & 3.4 & 0.032 & & \\
\hline \multicolumn{6}{|c|}{ SEQUENTIAL TESTS } \\
\hline Variable & $\mathrm{R}^{2}$ & Pseudo-F & Var\% & Cum\% & $\mathrm{P}$ \\
\hline $\mathrm{D}$ & 0.08 & 3.99 & 8.1 & 8.1 & 0.001 \\
\hline S & 0.16 & 3.98 & 7.6 & 15.7 & 0.001 \\
\hline Sand & 0.30 & 4.06 & 7.0 & 22.7 & 0.001 \\
\hline $\mathrm{BW} \delta^{15} \mathrm{~N}$ & 0.44 & 3.71 & 5.5 & 28.2 & 0.001 \\
\hline $\mathrm{BW} \mathrm{C} / \mathrm{N}$ & 0.49 & 3.70 & 5.1 & 33.3 & 0.001 \\
\hline $\mathrm{BW} \delta^{13} \mathrm{C}$ & 0.38 & 3.06 & 4.9 & 38.1 & 0.001 \\
\hline $\mathrm{T}$ & 0.20 & 2.44 & 4.5 & 42.7 & 0.001 \\
\hline Mud & 0.33 & 2.26 & 3.8 & 46.4 & 0.001 \\
\hline Gravel & 0.23 & 1.29 & 2.4 & 48.8 & 0.127 \\
\hline
\end{tabular}




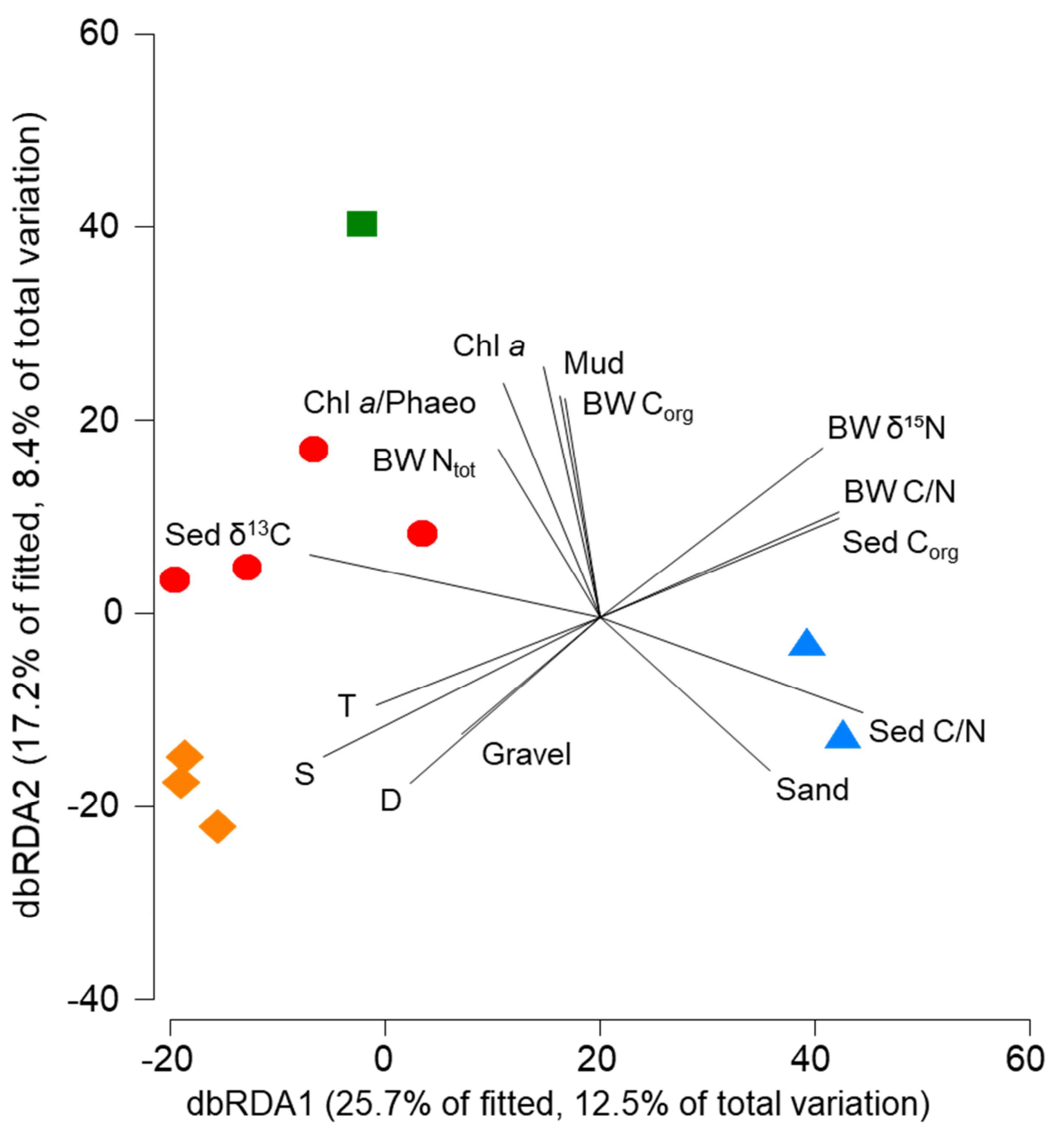

537 Fig. 5. Distance-based Redundancy Analysis (dbRDA) plot of the DistLM model visualizing

538 the relationships between the environmental parameters and the biomass/density ratio of

539 species between four sampling areas: A (Hornsund, Van Mijenfjorden); B (Storfjorden); C

540 (Barents Sea shelf); D (northern Barents Sea and Nansen Basin). Environmental variables

541 with Pearson rank correlations with dbRDA axes $>0.5$ are shown. Environmental factors: D -

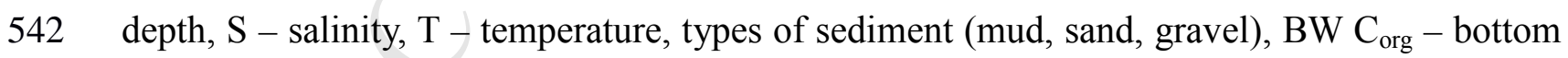

543 water $\mathrm{C}_{\text {org }}$, BW $\mathrm{N}_{\text {tot }}$ - bottom water $\mathrm{N}_{\text {tot }}, \mathrm{BW} \delta^{15} \mathrm{~N}$ - bottom water $\delta^{15} \mathrm{~N}, \mathrm{BW} \mathrm{C} / \mathrm{N}$ - bottom

544 water $\mathrm{C} / \mathrm{N}$, Sed $\mathrm{C}_{\text {org }}-\mathrm{C}_{\text {org }}$ concentration in sediment, Sed $\delta^{13} \mathrm{C}-$ sediment $\delta^{13} \mathrm{C}$, Sed $\mathrm{C} / \mathrm{N}-$

545 sediment $\mathrm{C} / \mathrm{N}, \mathrm{Chl} a$ - sediment Chlorophyll $a$ and $\mathrm{Chl} a / \mathrm{Phaeo}$ - sediment Phaeopigments. 
After 10 days, almost all luminophores ( 95\%) remained on sediment core surface at all sampling stations meaning that about $5 \%$ of luminophores were transported into sediments. The fastest decrease was noted at the B group (Storfjorden : AX/3 and ST/8), and at the C group (Southern Barents Sea station (AX/6) ; Nansen Basin < 400 m (PS/20, PS/32)) where $\sim 15$ to $25 \%$ of surface luminophores were buried. While luminophores were still present all 554 along the sedimentary column in the Storfjorden station, some subsurface peaks of 555 luminophores were clearly measured below $3 \mathrm{~cm}$ in the $\mathrm{C}$ group. The lowest decrease of the luminophores over depth was noted in the A group (Svalbard Fjords AX1/1, AX/2) and in the D group at deepest station (PS/31) in the Nansen Basin where 92 to $98 \%$ of luminophores remained at surface with slight subsurface peaks of tracers (about: only $0.91 \%$ ) between 1 to $5593 \mathrm{~cm}$ deep . Biodiffusion rates ranged from $0.04 \mathrm{~cm}^{-2} \mathrm{y}^{-1} \pm 0.01$ standard error (SE) (station AX/2) to $0.07 \mathrm{~cm}^{-2} \mathrm{y}^{-1} \pm 0.03 \mathrm{SE}$ (station $\mathrm{AX} / 1$ ) in group A. In group B biodiffusion rates was 0.06

$562 \mathrm{~cm}^{-2} \mathrm{y}^{-1} \pm 0.04 \mathrm{SE}$ (station $\mathrm{AX} / 3$ ). In group $\mathrm{C}$ biodiffusion ranged from 0 (station PS/32) to $0.76 \mathrm{~cm}^{-2} \mathrm{y}^{-1} \pm 0.71 \mathrm{SE}$ (station AX/6). There was no biodiffusive transport in group D. There 564 were significant differences in biodiffusion among areas (Kruskal-Wallis test; p<0.05; 565 significant differences (post hoc test) between group A:D and C:D) (Fig. 6A). Non-local 566 transport rates ranged from $0.21 \mathrm{y}^{-1} \pm 0.20 \mathrm{SE}$ (station $\mathrm{AX} / 2$ ) to $0.60 \mathrm{y}^{-1} \pm 0.23 \mathrm{SE}$ (station $567 \mathrm{AX} / 1$ ) in group A. In group B non-local transport rates was $2.12 \mathrm{y}^{-1} \pm 1 \mathrm{SE}$ (station AX/3). In 568 group C non-local transport rates ranged from $0.75 \mathrm{y}^{-1} \pm 0.25 \mathrm{SE}$ (station PS/32) to $2.08 \mathrm{y}^{-1} \pm$ 569 0.58 SE (station AX/6). In group D non-local transport rates ranged from $0.28 \mathrm{y}^{-1} \pm 0.04 \mathrm{SE}$ 570 (station PS/31) to $0.68 \mathrm{y}^{-1} \pm 0.31 \mathrm{SE}$ (station PS/19). There were significant differences in 571 non-local transport (Kruskal-Wallis test; $\mathrm{p}<0.05$; significant differences (post hoc test) 572 between group A:C and C:D) (Fig. 6B). Biodiffusive transport values were significantly 
573 related with depth, Sed $\mathrm{C}_{\text {org }}$ and BW C/N ratio Spearman correlation: -0.6, 0.6 and 0.6, $\mathrm{p}<0.05$

574 respectively). Non-local transport values were significantly related to benthic taxa richness,

575 biomass, mud and Sed $\mathrm{N}_{\text {tot }}$ (Spearman correlation: $0.5,0.5,0.5$ and $0.5 \mathrm{p}<0.05$ respectively)

576 (Table 8).

$H(3, N=47)=20.4\left(A\right.$ vs $D^{* *} ; C$ vs $\left.D^{* *}\right)$

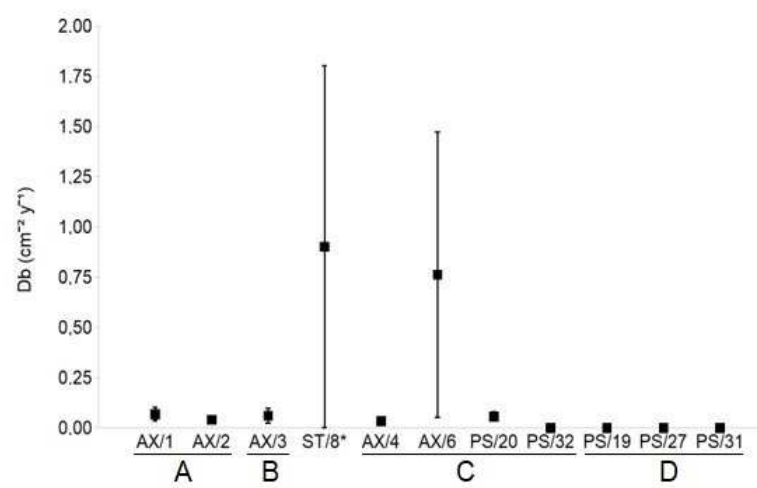

A

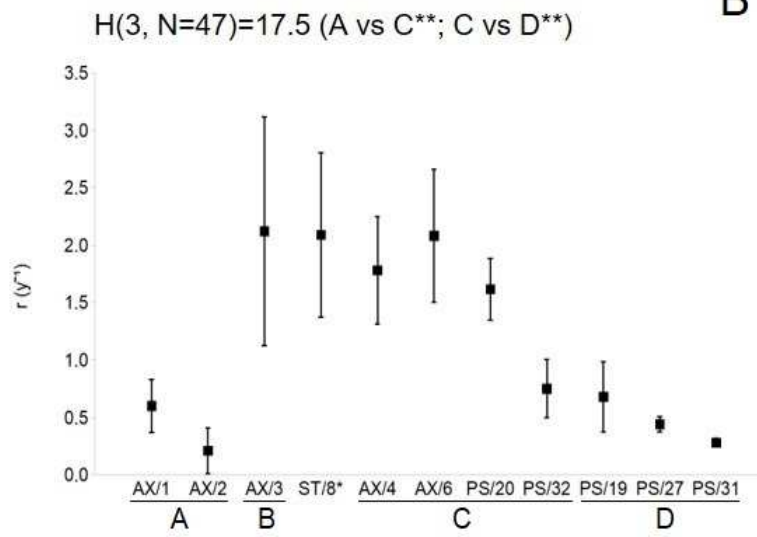

578 Fig. 6. Mean bioturbation coefficients: $\mathrm{Db}$ - biodiffusion $\left(\mathrm{cm}^{-2} \mathrm{y}^{-1}\right)(\mathrm{A})$ and $\mathrm{r}-$ non-local $\left(\mathrm{y}^{-1}\right)$

579 (B); \pm SE, n=no of cores (Table 1) at stations sampled in Van Mijenfjorden, Hornsund (group

580 A); Storfjorden (group B); Barents Sea shelf (group C); northern Barents Sea and Nansen

581 Basin (group D). Station ST/8 marked with * was sampled in summer season. Kruskal -

582 Wallis results for differences between sampling sites are given; significant test results are

583 marked with $* *(\mathrm{p}<0.05)$. 
Table 8. Spearman's rank correlation analyses among biological and physical parameters. Significant values are marked in bold ( $\mathrm{p}<0.05)$.

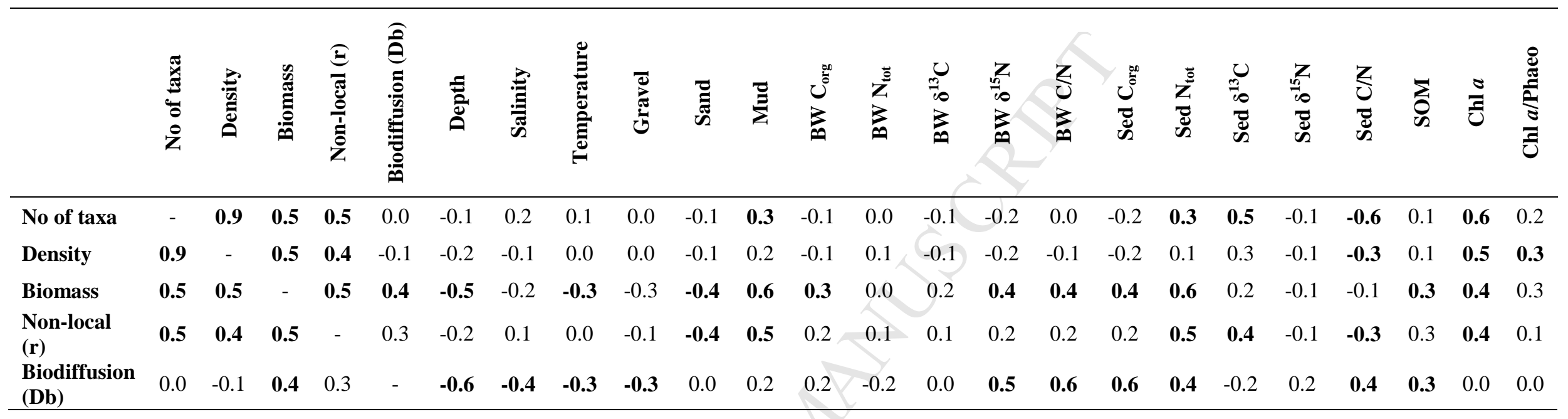


Bottom water salinity was similar in spring and summer in Storfjorden, respectively 34.5 and 34.1. Bottom water temperature in spring season was $-0.8{ }^{\circ} \mathrm{C}$ and increased to $4.5^{\circ} \mathrm{C}$

(Table 1). Benthic density decreased from 3625.3 ind. $/ \mathrm{m}^{2} \pm 83.9 \mathrm{SE}$ in spring $(\mathrm{AX} / 3)$ to

1812.7 ind. $/ \mathrm{m}^{2} \pm 229.7 \mathrm{SE}$ in summer (ST/8). Biomass was similar in both seasons (172.3

$\mathrm{g} / \mathrm{m}^{2} \pm 37.3 \mathrm{SE}$ (spring, AX/3) and $152.1 \mathrm{~g} / \mathrm{m}^{2} \pm 67.5 \mathrm{SE}$ (summer, ST/8). Non-local transport

rates were similar in spring and summer $\left(2.12 \pm 1\right.$ and $2.09 \pm 0.72 \mathrm{y}^{-1}$ respectively) but biodiffusion rates increased in summer $\left(0.06 \pm 0.04\right.$ in spring and $0.90 \pm 0.90 \mathrm{~cm}^{2} \mathrm{y}^{-1}$ in between spring and summer seasons (Mann-Whitney $U$-test; $Z=2.3 ; p<0.05$ and $Z=2.4$; $\mathrm{p}<0.05$, respectively).

598

\section{Discussion}

600

This is the first complex report on bioturbation activities in spring to summer transition time conducted over the large area from Svalbard fjords and Barents Sea to deep basin north off Svalbard. In our study, benthic community variables differentiated four groups of stations, and this separation was to some extent echoed by the environmental

605 factors. The benthic community properties further affected the measured benthic activities i.e. 606 bioturbation rates.

607 
The structure and composition of the benthic communities seemed to be grouped in

611 four groups of stations, however, the primary variables of the benthic community structure

612 (e.g., density, biomass) were highly variable within each station. One of the reasons could be

613 the high variability among replicates that covered only small area of sampled sediment.

614 Benthic species richness of the region was not well represented in the cores taken for the

615 experiments at each station due to their small sampling unit $\left(\sim 113 \mathrm{~cm}^{2}\right)$. This may have

616 implications for the results generalization to the sampled areas due to high variability of

617 benthic density, biomass and consequently on the effects of these on the measured

618 bioturbation coefficients. However, we assume that we collected the most abundant taxa,

619 which would likely have the dominant role in mediating bioturbation effect. We have sampled

620 and identified the benthic organisms in each sediment core where the bioturbation

621 experiments were processed meaning that we know the organisms responsible of the

622 measured bioturbation activities.

The variability of the quality and quantity of sedimentary OM within each station

624 impacted benthic community structure. For instance, the lowest quality of SOM (C/N 18)

625 was found in sandy sediments of Van Mijenfjorden and Hornsund fjords, highlighting

626 dominance of refractory organic material within the group A. This probably explains the

627 lowest benthic densities and biomass at these stations. This can be the result of geographical

628 locations (e.g., water circulation restrictions) and glacial activity in Hornsund, related high

629 sedimentation rates and high terrestrial OM inputs disturbing benthic fauna (Drewnik et al.,

630 2016; Włodarska-Kowalczuk and Pearson, 2004). These two stations’ communities were

631 mainly shaped by presence of polychaete Polycirrus arcticus and cumacean Diastylis lucifera,

632 and opportunistic cirratulids were among dominants.

633 A high, but variable, B/D ratio was found in Storfjorden (group B), which is the site of 634 a recurring polynya and has some of the highest productivity in the Barents Sea. The low 
635 temperature, presence of a sea ice-edge bloom, very fine grained sediments (muds) indicating

636 low hydrodynamism, and high $\mathrm{C}_{\text {org }}$ and $\mathrm{Chl} a$ contents in sediments and in bottom waters

637 promote diverse and high biomass benthic communities with larger individuals burrowing and

638 feeding deeper (Winkelmann and Knies, 2005). High Chl $a /$ Phaeo ratio and large quantities of

639 Chl $a$ within sediments show also a more frequent input of fresh OM because of the presence

640 of the polynya (Haarpaintner et al., 2001; Vinje, 2001; Winkelman and Knies, 2005). This

641 community was also diverse, with high dominance of polychaetes (cirratulids, carnivore

642 Lumbrineris sp, and subsurface tube-building conveyor Maldane sarsi) and bivalves like

643 Yoldia hyperborea and Nuculana radiata. PCO indicated that samples collected in Storfjorden

644 were correlated with presence of bivalve Ennucula tenuis and polychaete Apistobanchus 645 tulbergi.

646 The group C, covering stations sampled over the Barents Sea shelf, was highly 647 variable in terms of community structure and various environmental factors. The southern 648 Barents Sea station (AX/6) was characterized by similar environmental conditions to the 649 Storfjorden resulting in the occurrence of fauna with similar characteristics but with a lower 650 biomass to density ratio. This station was dominated by tube-building conveyor polychaete 651 Spiochaetopterus typicus and Heteromastus sp. and bivalve Macoma sp. It was characterized 652 by high amount of SOM but low Chl $a$ in the sediments, indicating late bloom/post bloom 653 conditions, where most of the fresh OM was already utilized by benthic organisms. Lower 654 Chl $a$ /Phaeo ratio also indicates more degraded OM, possibly a result of intensive pelagic 655 grazing (Morata and Renaud, 2008). This is likely since this station is in the southernmost 656 location, therefore it was under the strong influence of Atlantic waters and was already in the 657 late-phase of the phytoplankton bloom (Krause et al., 2018). The other stations from this 658 group, located in the northern part of Barents Sea e.g. Erik Eriksen Strait, were characterized 659 by high share of sand but relatively fresh and abundant SOM, though low Chl $a$ levels. This is 
660

661

662

663

664

665

666

667

668

669

670

671

672

673

674

675

676

677

678

679

680

681

682

683

684

quite similar to stations north of Svalbard (PS/20 and PS/32), although the later was characterized by higher amounts of $\mathrm{Chl} a$ present in the sediment related to the bloom in progress at the time of sampling (Peeken, 2016). This group was characterized by highly diverse communities (from 36 (AX/6) to 68 (PS/32) number of taxa), however most of them belonged to either biodiffusors or conveyors.

The northern Barents Sea and Nansen Basin (group D) were differentiated from the other groups by salinity, temperature, depth and occurrence of gravel. The species shaping communities included polychaetes Prionospio cirrifera and Notoproctus oculatus. Stations in group D were seasonally ice covered (including during sampling), and deeper than $400 \mathrm{~m}$. They were characterized by low amount of Sed $\mathrm{C}_{\text {org }}$ but presence of high OM quality in bottom water layer, perhaps indicating recent sedimentation. They were characterized by much lower benthic biomass and higher benthic density which were both decreasing with increasing depth regardless the bloom stage. Decreasing quality and quantity of OM with increasing depth, as it was observed in Nansen Basin, are often reflected in spatial variations in benthic community structure (Carroll et al., 2008) and result in severe energy limitation for deep-seafloor communities, dominated by small sized-individuals in high densities (Gage and Tyler, 1991; Bergmann et al., 2009; Grebmeier et al., 1988; Renaud et al., 2008).

Long living Arctic benthic fauna reflects carbon export fluxes to the sea bottom but changes are observed after several years or decades at deep stations (Grebmeier, 2012; Link et al., 2013). Therefore, it is likely that the bloom stage and current OM delivery to the sea floor did not directly influence the benthic community structure which is mainly dependent on the integrated carbon input to the sediments and OM pool available in the sediment. However, fresh OM arriving to the sea floor can trigger fast benthic response in terms of feeding and related movement (Morata et al., 2015; Boetius et al., 2013), thus influence the community functioning. 

hyperbaric conditions. Although, Glud et al. (1994) showed that in situ measurements of sediment oxygen demand/oxygen penetration depth were uniformly higher than deck

691 incubations, the relative differences among stations did not change with depth. Since other published studies have also not incorporated hyperbaric chambers when estimating sediment oxygen demand (Boetius et al., 2013), metabolism (Linke et al., 1995), and bioturbation (Clough et al., 1997), our results, as the relative rates, remain comparable. quantified in the stations of groups $\mathrm{A}, \mathrm{B}$ and $\mathrm{C}$ where a higher diversity of bioturbation groups was recorded among the dominant taxa (Table 4). Stations of the group D, on the contrary, exhibited lower diversity of functional traits among the dominant taxa with the simultaneous presence of two groups of sediment mixing (conveyors, biodiffusers) but where only non$700 \quad$ local transport was observed.

Species identity and differences in species characteristics, such as feeding mode and typical burrowing depth, have been previously shown to influence the intensity of bioturbation (Viitasalo-Frösén et al., 2009; Josefson et al., 2012; Näkki et al., 2017) and functional diversity can be considered to have more impacts than taxonomic richness (Harvey et al., 2012; Link et al., 2013). However, interpreting the bioturbation processes and the assignment of the macrofaunal species to the correct functional group can be challenging.

707 Short-term experimental studies of sediment mixing provide just essential data for only few species and results are hard to compare with longer time-scale processes in natural 
710

sediment mixing groups across their ranges since species are known to be able to feed in several different ways and change their feeding and mobility to exploit the food resources available (Biles et al., 2002). Change in organism feeding behavior will also mean a change in bioturbation which is reduced as a result of decreasing species diversity and community biomass, as well as diversity of feeding and bioturbation groups (Mazik and Elliott, 2000). In general, in our study, the high number of sub-surface deposit feeders, which feed at depth and transport material to the surface can explain dominance of non-local mixing (Boudreau, 1997; Gérino et al., 1998). Conveyors were actually omnipresent and the intensity of non-local transport was variable between stations, and was increasing with increasing species richness, density and biomass, but also with increasing percentage of mud presence and Chl $a$ contents (Table 8). Since non-local transport is non-continuous, it is usually difficult to link it directly to benthic biomass (or biovolume) since some movements of particles do not depend only on animal movements, but also on animal-independent effects (e.g., particles falling down into the burrows, initial burrows construction). In our study, increasing benthic biomass generated by high labile OM inputs, seems to have positive effects on the conveying activities and consequently on the non-local transport rates.

In shallow fjords (Van Mijenfjorden and Hornsund, Group A), the benthic communities were characterized by low bioturbation rates (non-local transport from $0.21 \pm$ 0.20 to $0.60 \pm 0.23 \mathrm{y}^{-1}$ and biodiffusion from $0.04 \pm 0.01$ to $0.07 \pm 0.03 \mathrm{~cm}^{-2} \mathrm{y}^{-1}$ ). This similarity between the $\mathrm{AX} / 1$ and $\mathrm{AX} / 2$ stations of the group $\mathrm{A}$ can be explained by the occurrence of a similar benthic community whose the species have the same functional traits combination (i.e., feeding, mobility, burrowing depth, burrowing mode and sediment mixing mode; Table 4) but present in low density and biomass. Biodiffusors (Cirratulidae; Gérino et al., 1992, 2007), conveyors (P. arcticus) and gallery diffusors (Lumbrineris sp.; Petch, 1986), 
734 both present in those fjords, were probably limited by the lack of fresh OM in the sediments at 735 the time of sampling.

737 similar measured bioturbation rates with high non-local transport and low biodiffusion, and 738 shared a high biomass contribution of bivalves and maldanid polychaetes. Yoldiids and 739 maldanids, burrowing deeper into the sediment, are known to be effective conveyors which 740 can either actively transfer sediment directly into deep layers from the surface, or into surface 741 layers from deeper layers of the sediments (Bender and Davis, 1984; Smith and Schafer, 742 1984), respectively. This can explain high non-local transport in these areas. $\mathrm{Db}$ and $\mathrm{r}$ by station within the group, with a relatively higher biomass and density of the organisms (Spiochaetopterus typicus (conveyor, Smith and Shafer, 1984) dominating in the density and Astarte sp. (biodiffusor, Queirós at al., 2013) dominating in the biomass. The reason for that was most likely earlier occurrence of the phytoplankton bloom due to the lack of sea ice, so at the time of the cruise, late spring/post-bloom conditions and abundant OM activated rapidly benthic organisms.

All deeper and sea ice covered stations in group D, including sea ice covered PS/32

751 (group C), were dominated by biodiffusors and conveyors (Table 4). Those stations were 752 characterized by significant non-local transport and unmeasurable biodiffusion. This suggests 753 that the sediment transport mode by conveyors dominated in the sampled deeper areas 754 adapted to scarce fresh food availability despite the presence of biodiffusers. This implies a 755 very low activity by biodiffusers undetectable at the time scale of 10 days of experiment, or 756 an interfering of such activities with the conveyors species. Typically for communities living 757 in OM limited environments, these benthic communities were characterized by a low number 758 of species with low biomass. Giving the low bioturbation rates in the deeper stations, we 
could actually suppose that time incubation with luminophores superior to 10 days could be tested in the future experiments in order to insure a more complete transport of tracers towards deep layers where the benthic community is less active. Since the bioturbation model takes into account in its calculation this time duration, the final bioturbation coefficient is however normalized to this time scale, signifying the similar relative comparison between stations for our experiment remains valid. If we were able to detect measurable biodiffusive coefficient for the deeper stations for a longer time scale, normalized to the same time unit, the biodiffusion would be still low because of weak benthic infauna dynamic in such environments. Our results are, however, similar to the patterns showed by Clough et al. (1997) who noted low biodiffusive transport $\left(0.01\right.$ to $\left.0.11 \mathrm{~cm}^{-2} \mathrm{y}^{-1}\right)$ by using radioactive tracer for its deep portions in relationships to the lower benthic biomass due to lower fresh OM inputs. They found, however, higher surficial sediment biological mixing rates than the natural sedimentation rates in the Arctic deep sediments, highlighting the importance of quantifying bioturbation in the Arctic Ocean taking into account its spatial variability. Also, Soltwedel et al. (2019), using luminophores for experiments that lasted 2 and 4 years, found low biodiffusion mixing rates at the Long-Term Ecological Research (LTER) observatory HAUSGARTEN in Fram Strait $\left(\sim 0.2 \mathrm{~cm}^{-2} \mathrm{y}^{-1}\right)$. They concluded that the meiofauna, and to a certain extent megafauna, were the main bioturbators in the deep sea environments. Although, only macrofauna $(>500 \mu \mathrm{m})$ was identified and was presented as the main responsible of the bioturbation activities in our study, meiofaunal activities may also explain some of the patterns that could not be attributed to the changes in the environment or macrofaunal communities in the deep sea stations. Since the model quantifies bioturbation coefficients of the entire benthic community, it includes here also the meiofauna activities. Meiofauna is actually known as the most abundant infauna (Heip et al., 1985; Vanreusel et al., 2010; Rosli et al., 2016; own data) and as having impact on the bioturbation activities in the first few 
784 centimeters of the sediment (Aller and Aller, 1992; Piot el al., 2014; Aschenbroich et al.,

785 2017; Mäkelä et al., 2018). Therefore, we suggest that also the smaller benthic fractions

786 should be identified in future studies on Arctic deep infaunal activity in order to find better

787 infauna variables for explaining the bioturbation measurements.

788

\subsection{Seasonal changes (Storfjorden station)}

790

791

792

793

794

795

796

797

798

799

800

801

802

803

804

805

806

807

808

The highest values of non-local transport were reported for Storfjorden and remained stable during both spring and summer time. The biodiffusion coefficient measured in Storfjorden during summer was about fifteen times higher compared to measurements conducted during spring, probably because of the changes in species and functional groups between the two seasons associated with the more labile OM reaching the seafloor in summer (Chl a/Phaeo 52, Table 3). The dominant taxa in density were Cirratullidae (biodiffusor) both during spring and summer (31.2\% and $11 \%$, respectively) and Lumbrineris sp. (gallery diffusor) (14.1\% and $18.3 \%$, respectively) while in biomass, Yoldia hyperborea (convoyer) dominated during both seasons $(57.4 \%$ and $30.5 \%$, respectively) (Table 4). Such changes, i.e. replacement of one large specimen by another large specimen of different species, should be rather accounted to spatial variability of benthic communities than due to seasonal change in sampled communities. Also, species such as Nereis diversicolor or M. balthica are able to change their feeding mode (suspensive-feeder versus deposit feeder) depending on environmental conditions (De Goej and Luttikhuizen, 1998; Christensen et al., 2000). It is also possible that taxa such as Cirratulidae, Lumbrinereis, Yoldia and Nuculana change their feeding mode with increased OM input from the polynya during the summer just after the spring bloom, as was observed in other ecosystems or experiments (e.g., Bender and Davis, 1984 for Yoldia spp; Rouse and Pleijel, 2001; Kędra et al., 2012 for Cirratulidae). 
Benthic activities are related to the supply of OM to the seabed (Grassle and Grassle,

810 1994; Levin and Gooday, 2003; Blake et al., 2009) and biodiffusion intensity can be strongly

811 dependent on flux of fresh food from overlying waters (Gérino et al., 1998). In shallow

812 sediments of temperate areas, Duport et al. (2007) found the highest intensity of sediment

813 mixing (non-local and biodiffusion) during summer in the Thau Lagoon. Also Gérino et al.

814 (2007) found more rapid non-local transport in spring than in autumn in the Venice Lagoon.

815 Organic carbon supply generally increases when the PP starts in spring, and peaks with the

816 bloom and afterwards, over the summer. In the polar areas, Morata et al. (2015) found

817 minimum biodiffusive activity during the polar night, and high non-local transport after a

818 pulse of fresh food in experiments conducted in high Arctic fjord in Svalbard. This result

819 suggests that behavior of benthic species change with the OM input. Also, laboratory

820 experiments showed that macrofauna can react quickly to food input by increasing their

821 bioturbation activities (Nogaro et al., 2008). Furthermore, Dauwe et al. (1998) reported

822 maximum sediment mixing with medium food quality in the study comparing macrofaunal

823 benthic activity with contrasting food supply in the North Sea. They also showed that the

824 minimal mixing was observed at the station with high quality OM, and no mixing when low

825 quality OM was present. This can result from combination of physical and biochemical 826 factors influencing bioturbation, or changes in species behavior. The other possible 827 explanation is related to the oxygen conditions in different areas. Both North and Baltic Sea 828 are areas with high PP and eutrophication. Higher amounts of new OM reaching the sea floor 829 often result in low oxygen levels leading to anoxic conditions affecting faunal behavior or 830 even survival (Carstensen et al., 2014). Svalbard fjords and adjacent areas are largely oligo831 and meso- trophic, and well oxygenated regardless the time of the year and intensity of the 832 spring bloom deposition. We, thus, expect minimal negative impacts of OM deposition on 
833 benthic communities. Our results from this limited seasonal comparison suggest that large

834 inputs of fresh OM to the seabed can trigger bioturbation activities.

835

836

837

838

839

840

841

842

843

844

845

846

847

848

849

850

851

852

853

854

855

856

857

\section{Acknowledgements}

This work is a contribution to the Polish National Science Centre (project number 2015/19/B/NZ8/03945 (SeaIceFun) to MK) and the Norwegian Research Council (ARCEx project \#228107). We would like to thank the crew and scientific team for support and assistance at the sea during the Arctic in Rapid Transition (ART) Network cruise TRANSSIZ (ARK XXIX/1; PS92) on R/V Polarstern, ARCEx cruise on R/V Helmer Hanssen and SteP cruise on R/V L'Atalante, especially to Maeve McGovern. We would like to thank Leading National Research Centre (KNOW) received by the Centre for Polar Studies for the financial support. Thank you to Aleksandra Winogradow (IOPAN) and Jeremy Devesa (LEMAR) for their help during CHN analyses. Mobility grants of BO, NM and EM were supported by the French National Agency under the program "Investissements d'Avenir" (LabexMER: ANR10-LABX-19) and by the scientific committee of IUEM to EM. We thank three anonymous reviewers for their comments, which significantly improved the paper. This paper is a Nereis Park number 41.

\section{References:}

Aller, R.C., Aller, J.Y., 1992. Meiofauna and solute transport in marine muds. Limnology and Oceanography 37, 1018-1033. https://doi.org/10.4319/lo.1992.37.5.1018

Anderson, M.J., Gorley, R.N., Clarke, K.R., 2008. PERMANOVA for PRIMER: guide to software and statistical methods. PRIMER-E Ltd., Plymouth, United Kingdom p. 214.

Andreassen, I., Wassmann, P., 1998. Vertical flux of phytoplankton and particulate biogenic matter in the marginal ice zone of the Barents Sea in May 1993. Marine Ecology 
859

860

861

862

863

864

865

866

867

868

869

870

871

872

873

874

875

876

877

878

879

880

881

882

Aschenbroich, A., Michaud, E., Gilbert, F., Fromard, F., Alt, A., Le Garrec, V., Bihannic, I., De Coninck, A., Thouzeau, G., 2017. Bioturbation functional roles associated with mangrove development in French Guiana, South America. Hydrobiologia 794, 179202. https://doi.org/10.1007/s10750-017-3093-7

Bender, K., Davis, W.R., 1984. The effect of feeding by Yoldia limatula on bioturbation. Ophelia 23, 91-100. https://doi.org/10.1080/00785236.1984.10426606

Bergmann, M., Dannheim, J., Bauerfeind, E., Klages, M., 2009. Trophic relationships along a bathymetric gradient at the deep-sea observatory HAUSGARTEN. Deep Sea Research Part I: Oceanographic Research Papers 56, 408-424. https://doi.org/10.1016/j.dsr.2008.10.004

Berner, R.A., 1980. Early diagenesis. A theoretical approach. Princeton University Press, Princeton, NJ.

Biles, C.L., Paterson, D.M., Ford, R.B., Solan, M., Raffaelli, D.G., 2002. Bioturbation, ecosystem functioning and community structure. Hydrology and Earth System Sciences 6, 999-1005. https://doi.org/10.5194/hess-6-999-2002

Blake, J.A., Maciolek, N.J., Ota, A.Y., Williams, I.P., 2009. Long-term benthic infaunal monitoring at a deep-ocean dredged material disposal site off Northern California. Deep Sea Research Part II: Topical Studies in Oceanography 56, 1775-1803. https://doi.org/10.1016/j.dsr2.2009.05.021

Błaszczyk, M., Jania, J.A., Kolondra, L., 2013. Fluctuations of tidewater glaciers in Hornsund Fjord (Southern Svalbard) since the beginning of the 20th century. Polish Polar Research 34, 327-352. https://doi.org/10.2478/popore-2013-0024

Blott, S.J., Pye, K., 2001. GRADISTAT: a grain size distribution and statistics package for the analysis of unconsolidated sediments. Earth Surface Processes and Landforms 26, 
883

884

885

886

887

888

889

890

891

892

893

894

895

896

897

898

899

900

901

902

903

904

905

906

907

1237-1248. https://doi.org/10.1002/esp.261

Boetius, A., Albrecht, S., Bakker, K., Bienhold, C., Felden, J., Fernandez-Mendez, M., Hendricks, S., Katlein, C., Lalande, C., Krumpen, T., Nicolaus, M., Peeken, I., Rabe, B., Rogacheva, A., Rybakova, E., Somavilla, R., Wenzhofer, F., RV Polarstern ARK273-Shipboard Science Party, 2013. Export of Algal Biomass from the Melting Arctic Sea Ice. Science 339, 1430-1432. https://doi.org/10.1126/science.1231346

Bouchet, V.M.P., Sauriau, P.-G., Debenay, J.-P., Mermillod-Blondin, F., Schmidt, S., Amiard, J.-C., Dupas, B., 2009. Influence of the mode of macrofauna-mediated bioturbation on the vertical distribution of living benthic foraminifera: First insight from axial tomodensitometry. Journal of Experimental Marine Biology and Ecology 371, 20-33. https://doi.org/10.1016/j.jembe.2008.12.012

Boudreau, B.P., 1986. Mathematics of tracer mixing in sediments. 2. Nonlocal mixing and biological conveyor-belt phenomena. American Journal of Science 286, 199-238.

Boudreau, B.P., 1997. Diagenetic models and their implementation. Springer Verlag, 414 pp.

Bourgeois, T., Orr, J.C., Resplandy, L., Terhaar, J., Ethé, C., Gehlen, M., Bopp, L., 2016. Coastal-ocean uptake of anthropogenic carbon. Biogeosciences 13, 4167-4185. https://doi.org/10.5194/bg-13-4167-2016

Carroll, J., Zaborska, A., Papucci, C., Schirone, A., Carroll, M.L., Pempkowiak, J., 2008. Accumulation of organic carbon in western Barents Sea sediments. Deep Sea Research Part II: Topical Studies in Oceanography 55, 2361-2371. https://doi.org/10.1016/j.dsr2.2008.05.005

Carstensen, J., Andersen, J.H., Gustafsson, B.G., Conley, D.J., 2014. Deoxygenation of the Baltic Sea during the last century. Proceedings of the National Academy of Sciences 111, 5628-5633. https://doi.org/10.1073/pnas.1323156111

Christensen, B., Vedel, A., Kristensen, E., 2000. Carbon and nitrogen fluxes in sediment 

inhabited by suspension-feeding (Nereis diversicolor) and non-suspension-feeding (N. virens) polychaetes. Marine Ecology Progress Series 192, 203-217. https://doi.org/10.3354/meps192203

911 Clarke, K.R., Gorley, R.N., 2006. PRIMER v6: User manual/tutorial. PRIMER-E, Plymouth 912 UK.

913

914

915

916

917

918

919

920

921

922

923

924

925

926

927

Clough, L.M., Ambrose, W.G., Kirk Cochran, J., Barnes, C., Renaud, P.E., Aller, R.C., 1997. Infaunal density, biomass and bioturbation in the sediments of the Arctic Ocean. Deep Sea Research Part II: Topical Studies in Oceanography 44, 1683-1704. https://doi.org/10.1016/S0967-0645(97)00052-0

Cochrane, S.K.J., Pearson, T.H., Greenacre, M., Costelloe, J., Ellingsen, I.H., Dahle, S., Gulliksen, B., 2012. Benthic fauna and functional traits along a Polar Front transect in the Barents Sea - Advancing tools for ecosystem-scale assessments. Journal of Marine Systems 94, 204-217. https://doi.org/10.1016/j.jmarsys.2011.12.001

Codispoti, L.A., Kelly, V., Thessen, A., Matrai, P., Suttles, S., Hill, V., Steele, M., Light, B., 2013. Synthesis of primary production in the Arctic Ocean: III. Nitrate and phosphate based estimates of net community production. Progress in Oceanography 110, 126150. https://doi.org/10.1016/j.pocean.2012.11.006

D’Andrea, A.F., Lopez, G.R., Aller, R.C., 2004. Rapid physical and biological particle mixing on an intertidal sandflat. Journal of Marine Research 62, 67-92. https://doi.org/10.1357/00222400460744627

Dauwe, B., Herman, P.M.J., Heip, C.H.R., 1998. Communitv structure and bioturbation potential of macrofauna at four North Sea stations with contrasting food supply. Mar Ecol Prog Ser 173, 67-8317. https://doi:10.3354/meps173067

De Goeij, P., Luttikhuizen, P., 1998. Deep-burying reduces growth in intertidal bivalves: field and mesocosm experiments with Macoma balthica. Journal of Experimental Marine 
934 Drewnik, A., Węsławski, J.M., Włodarska-Kowalczuk, M., Łącka, M., Promińska, A., Zaborska, A., Gluchowska, M., 2016. From the worm's point of view. I: Environmental settings of benthic ecosystems in Arctic fjord (Hornsund, Spitsbergen). Polar Biology 39, 1411-1424. https://doi.org/10.1007/s00300-015-1867-9

Duchêne, J., Rosenberg, R., 2001. Marine benthic faunal activity patterns on a sediment surface assessed by video numerical tracking. Marine Ecology Progress Series 223, 113-119. https://doi.org/10.3354/meps223113

Duport, E., Gilbert, F., Poggiale, J.-C., Dedieu, K., Rabouille, C., Stora, G., 2007. Benthic macrofauna and sediment reworking quantification in contrasted environments in the Thau Lagoon. Estuarine, Coastal and Shelf Science 72, 522-533. https://doi.org/10.1016/j.ecss.2006.11.018

Fauchald, K., Jumars, P.A., 1979. The diet of worms: a study of polychaete feeding guilds. Oceanogr Mar Biol Annu Rev 17, 193-284.

Fernández-Méndez, M., Katlein, C., Rabe, B., Nicolaus, M., Peeken, I., Bakker, K., Flores, H., Boetius, A., 2015. Photosynthetic production in the central Arctic Ocean during the record sea-ice minimum in 2012. Biogeosciences 12, 3525-3549. https://doi.org/10.5194/bg-12-3525-2015

Fetzer, I., Lønne, O.J., Pearson, T., 2002. The distribution of juvenile benthic invertebrates in an Arctic glacial fjord. Polar Biol 25, 303-315.

Fisher, J.B., Lick, W.J., McCall, P.L., Robbins, J.A., 1980. Vertical mixing of lake sediments by tubificid oligochaetes. Journal of Geophysical Research 85 (C7), 3997-4006. https://doi.org/10.1029/JC085iC07p03997

François, F., Delegre, K., Gilbert, F., Stora G., 1999. Specific variability within functional groups. Study of the sediment reworking of two Veneridae bivalves, Ruditapes 
decussatus and Venerupis aurea. C. R. Acad. Sci. Paris, 322, 339-345.

959 François, F., Gérino, M., Stora, G., Durbec, J., Poggiale, J., 2002. Functional approach to sediment reworking by gallery-forming macrobenthic organisms: modeling and application with the polychaete Nereis diversicolor. Marine Ecology Progress Series 229, 127-136. https://doi.org/10.3354/meps229127

963

964

965

966

967

968

969

970

971

972

973

974

975

976

977

978

979

980

981

982

François, F., Poggiale, J.-C., Durbec, J.-P., Stora, G., 2001. A newmodel of bioturbation for a functional approach to sedi-ment reworking resulting from macrobenthic communities. In: Aller JY, Woodin SA, Aller RC (eds) Organism-sediment interactions. University of South Carolina Press,Columbia, p 73-86.

François, F., Poggiale, J.-C., Durbec, J.-P., Stora, G., 1997. A new approach for the modelling of sediment reworking induced by a macrobenthic community. Acta Biotheor. 45, 295319.

Gage, J., Tyler, P., 1991. Deep-Sea Biology: A Natural History of Organisms at the Deep-Sea Floor. Cambridge University Press, New York.

Gardner, L.R., Sharma, P., Moore, W.S., 1987. A regeneration model for the effect of bioturbation by fiddler crabs on $210 \mathrm{~Pb}$ profiles in salt marsh sediments. Journal of Environmental Radioactivity 5, 25-36.

Gérino, M., 1992. Étude expérimentale de la bioturbation en milieu littoral et profond. Doctoral dissertation, Université Aix-Marseille II. 196 pp.

Gérino, M., Aller, R.C., Lee, C., Cochran, J.K., Aller, J.Y., Green, M.A., Hirschberg, D., 1998. Comparison of Different Tracers and Methods Used to Quantify Bioturbation During a Spring Bloom: 234-Thorium, Luminophores and Chlorophylla. Estuarine, Coastal and Shelf Science 46, 531-547. https://doi.org/10.1006/ecss.1997.0298

Gérino, M., Frignani, M., Mugnai, C., Bellucci, L.G., Prevedelli, D., Valentini, A., Castelli, A., Delmotte, S., Sauvage, S., 2007. Bioturbation in the Venice Lagoon: Rates and 
relationship to organisms.

Acta Oecologica

32,

$14-25$. https://doi.org/10.1016/j.actao.2007.02.003

Gilbert, F., Hulth, S., Grossi, V., Poggiale, J.-C., Desrosiers, G., Rosenberg, R., Gérino, M., François-Carcaillet, F., Michaud, E., Stora, G., 2007. Sediment reworking by marine benthic species from the Gullmar Fjord (Western Sweden): Importance of faunal biovolume. Journal of Experimental Marine Biology and Ecology 348, 133-144. https://doi.org/10.1016/j.jembe.2007.04.015

Gingras, M.K., Pemberton, S.G., Dashtgard, S., Dafoe, L., 2008. How fast do marine invertebrates burrow? Palaeogeography, Palaeoclimatology, Palaeoecology 270, 280286. https://doi.org/10.1016/j.palaeo.2008.07.015

Glud, R.N., Gundersen, J.K., Barker Jørgensen, B., Revsbech, N.P., Schulz, H.D., 1994. Diffusive and total oxygen uptake of deep-sea sediments in the eastern South Atlantic Ocean:in situ and laboratory measurements. Deep Sea Research Part I: Oceanographic Research Papers 41, 1767-1788. https://doi.org/10.1016/0967-0637(94)90072-8

Gogina, M., Morys, C., Forster, S., Gräwe, U., Friedland, R., Zettler, M.L., 2017. Towards benthic ecosystem functioning maps: Quantifying bioturbation potential in the German part of the Baltic Sea. Ecological Indicators 73, 574-588. https://doi.org/10.1016/j.ecolind.2016.10.025

Gosselin, M., Levasseur, M., Wheeler, P.A., Horner, R.A., Booth, B.C., 1997. New measurements of phytoplankton and ice algal production in the Arctic Ocean. Deep Sea Research Part II: Topical Studies in Oceanography 44, 1623-1644. https://doi.org/10.1016/S0967-0645(97)00054-4

Grassle, J.F., Grassle, J.P., 1994. Notes from the abyss: the effects of a patchy supply of organic material and larvae on soft-sediment benthic communities. In: Giller, P.S., Hildrew, A.G., Raffacelli, D.G. (Eds.), Aquatic Ecology: Scale, Pattern and Process. 
Blackwell, Oxford, pp. 499-515.

1009 Grebmeier, J.M., 2012. Shifting Patterns of Life in the Pacific Arctic and Sub-Arctic Seas. Annual Review of Marine Science 4, 63-78. https://doi.org/10.1146/annurev-marine$\underline{120710-100926}$

1012

1013

1014

1015

1016

1017

1018

1019

1020

1021

1022

1023

1024

1025

1026

1027

1028

1029

1030

1031

1032

Grebmeier, J.M., Cooper, L.W., Feder, H.M., Sirenko, B.I., 2006. Ecosystem dynamics of the Pacific-influenced Northern Bering and Chukchi Seas in the Amerasian Arctic. Progress in Oceanography 71, 331-361. https://doi.org/10.1016/j.pocean.2006.10.001

Grebmeier, J.M, McRoy, C., Feder, H., 1988. Pelagic-benthic coupling on the shelf of the northern Bering and Chukchi Seas. I. Food supply source and benthic bio-mass. Marine Ecology Progress Series 48, 57-67. https://doi.org/10.3354/meps048057

Haarpaintner, J., Haugan, P.M., Gascard, J.-C., 2001. Interannual variability of the Storfjorden (Svalbard) ice cover and ice production observed by ERS-2 SAR. Annals of Glaciology 33, 430-436. https://doi.org/10.3189/172756401781818392

Harvey, E., Séguin, A., Nozais, C., Archambault, P., Gravel, D., 2013. Identity effects dominate the impacts of multiple species extinctions on the functioning of complex food webs. Ecology 94, 169-179. https://doi.org/10.1890/12-0414.1

Heip C, Vincx M, Vranken G. 1985. The ecology of marine nematodes. Oceanography and Marine Biology: An Annual Review 23, 399-489.

Holm-Hansen, O., Lorenzen, C.J., Holms, R.W., Strickland, J.D., 1965. Fluorometric determination of chlorophyll. J Conseil Int pour l'Exploration de la Mer 30, 3-15.

Jørgensen, L.L., Ljubin, P., Skjoldal, H.R., Ingvaldsen, R.B., Anisimova, N., Manushin, I., 2015. Distribution of benthic megafauna in the Barents Sea: baseline for an ecosystem approach to management. ICES Journal of Marine Science 72, 595-613. https://doi.org/10.1093/icesjms/fsu106

Josefson, A., Norkko, J., Norkko, A., 2012. Burial and decomposition of plant pigments in 
surface sediments of the Baltic Sea: role of oxygen and benthic fauna. Marine Ecology Progress Series 455, 33-49. https://doi.org/10.3354/meps09661

1035

1036

1037

1038

1039

1040

1041

1042

1043

1044

1045

1046

1047

1048

1049

1050

1051

1052

1053

1054

1055

1056

1057

Kędra, M., Kuliński, K., Walkusz, W., Legeżyńska, J., 2012. The shallow benthic food web structure in the high Arctic does not follow seasonal changes in the surrounding environment. Estuarine, Coastal and Shelf Science 114, 183-191. https://doi.org/10.1016/j.ecss.2012.08.015

Kędra, M., Pabis, K., Gromisz, S., Węsławski, J.M., 2013. Distribution patterns of polychaete fauna in an Arctic fjord (Hornsund, Spitsbergen). Polar Biology 36, 1463-1472. https://doi.org/10.1007/s00300-013-1366-9

Kennedy, P., Kennedy, H., Papadimitriou, S., 2005. The effect of acidification on the determination of organic carbon, total nitrogen and their stable isotopic composition in algae and marine sediment. Rapid Communications in Mass Spectrometry 19, 10631068. https://doi.org/10.1002/rcm.1889

Knaust, D., Bromley, R.G., 2012. Trace fossils as indicators of sedimentary environments. Developments in sedimentology, vol 64. Elsevier, Amsterdam.

Konovalov, D., Renaud, P.E., Berge, J., Voronkov, A.Y., Cochrane, S.K.J., 2010. Contaminants, benthic communities, and bioturbation: potential for PAH mobilisation in Arctic sediments. Chemistry and Ecology 26, 197-208. https://doi.org/10.1080/02757541003789058

Krause, J.W., Duarte, C.M., Marquez, I.A., Assmy, P., Fernández-Méndez, M., Wiedmann, I., Wassmann, P., Kristiansen, S., Agustí, S., 2018. Biogenic silica production and diatom dynamics in the Svalbard region during spring. Biogeosciences Discussions 1-25. https://doi.org/10.5194/bg-2018-226

Kristensen, E., Penha-Lopes, G., Delefosse, M., Valdemarsen, T., Quintana, C., Banta, G., 2012. What is bioturbation? The need for a precise definition for fauna in aquatic 


\section{ACCEPTED MANUSCRIPT}

1058

1059

1060

1061

1062

1063

1064

1065

1066

1067

1068

1069

1070

1071

1072

1073

1074

1075

1076

1077

1078

1079

1080

1081

1082

sciences. Marine Ecology

Progress

Series

446 ,

$285-302$.

https://doi.org/10.3354/meps09506

Kure, L.K., Forbes, T.L., 1997. Impact of bioturbation by Arenicola marina on the fate of particle-bound fluoranthene. Marine Ecology Progress Series 156, 157-166.

Leu, E., Wiktor, J., Søreide, J., Berge, J., Falk-Petersen, S., 2010. Increased irradiance reduces food quality of sea ice algae. Marine Ecology Progress Series 411, 49-60. https://doi.org/10.3354/meps08647

Levin, L.A., Gooday, A.J., 2003. The Deep Atlantic Ocean Chapter 5. In: Tyler, P.A. (Ed.), Ecosystems of the Deep Oceans. Ecosystems of the World 28. Elsevier, Amsterdam, pp. 111-178.

Link, H., Piepenburg, D., Archambault, P., 2013. Are Hotspots Always Hotspots? The Relationship between Diversity, Resource and Ecosystem Functions in the Arctic. PLoS ONE 8, e74077. https://doi.org/10.1371/journal.pone.0074077

Linke, P., Altenbach, A.V., Graf, G., Heeger, T., 1995. Response of deep-sea benthic foraminifera to a simulated sedimentation event. Journal of Foraminiferal Research 25, 75-82. https://doi.org/10.2113/gsjfr.25.1.75

Maire, O., Duchêne, J.C., Grémare, A., Malyuga, V.S., Meysman, F.J.R., 2007. A comparison of sediment reworking rates by the surface deposit-feeding bivalve Abra ovata during summertime and wintertime, with a comparison between two models of sediment reworking. Journal of Experimental Marine Biology and Ecology 343, 21-36. https://doi.org/10.1016/j.jembe.2006.10.052

Maiti, K., Carroll, J., Benitez-Nelson, C.R., 2010. Sedimentation and particle dynamics in the seasonal ice zone of the Barents Sea. Journal of Marine Systems 79, 185-198. https://doi.org/10.1016/j.jmarsys.2009.09.001

Mäkelä, A., Witte, U., Archambault, P., 2018. Short-term processing of ice algal- and 
1083

1084

1085

1086

1087

1088

1089

1090

1091

1092

1093

1094

1095

1096

1097

1098

1099

1100

1101

1102

1103

1104

1105

1106

1107

phytoplankton-derived carbon by Arctic benthic communities revealed through isotope labelling experiments. Marine Ecology Progress Series 600, 21-39. https://doi.org/10.3354/meps12663

Mazik, K., Elliott, M., 2000. The effects of chemical pollution on the bioturbation potential of estuarine intertidal mudflats. Helgoland Marine Research 54, 99-109. https://doi.org/10.1007/s101520050008

McClintic, M.A., DeMaster, D.J., Thomas, C.J., Smith, C.R., 2008. Testing the FOODBANCS hypothesis: Seasonal variations in near-bottom particle flux, bioturbation intensity, and deposit feeding based on 234Th measurements. Deep Sea Research Part II: Topical Studies in Oceanography 55, 2425-2437. https://doi.org/10.1016/j.dsr2.2008.06.003

McMeans, B.C., McCann, K.S., Humphries, M., Rooney, N., Fisk, A.T., 2015. Food Web Structure in Temporally-Forced Ecosystems. Trends in Ecology \& Evolution 30, 662672. https://doi.org/10.1016/j.tree.2015.09.001

McMinn, A., Pankowskii, A., Ashworth, C., Bhagooli, R., Ralph, P., Ryan, K., 2010. In situ net primary productivity and photosynthesis of Antarctic sea ice algal, phytoplankton and benthic algal communities. Marine Biology 157, 1345-1356. https://doi.org/10.1007/s00227-010-1414-8

Mermillod-Blondin, F., Marie, S., Desrosiers, G., Long, B., de Montety, L., Michaud, E., Stora, G., 2003. Assessment of the spatial variability of intertidal benthic communities by axial tomodensitometry: importance of fine-scale heterogeneity. Journal of Experimental Marine Biology and Ecology 287, 193-208. https://doi.org/10.1016/S0022-0981(02)00548-8

Meysman, F.J.R., Boudreau, B.P., Middelburg, J.J., 2003. New developments in the modelling of bioturbation in aquatic sediments: relations between local, non-local, discrete and 
continuous models. Terramare 12, 91-93.

1109 Michaud E., 2006. Effet of the Functional ecology of Macoma balthica community (St Lawrence estuary, Quebec, Canada) on biogeochemical fluxes at the sediment-water interface and on sediment mixing. PhD Thesis. University of du Québec à Rimouski et Université de la Méditerranée, Aix-Marseille II, 237p.

Michaud, E., Desrosiers, G., Mermillod-Blondin, F., Sundby, B., Stora, G., 2005. The functional group approach to bioturbation: The effects of biodiffusers and gallerydiffusers of the Macoma balthica community on sediment oxygen uptake. Journal of Experimental Marine Biology and Ecology 326, 77-88. https://doi.org/10.1016/j.jembe.2005.05.016

Michaud, E., Desrosiers, G., Mermillod-Blondin, F., Sundby, B., Stora, G., 2006. The functional group approach to bioturbation: II. The effects of the Macoma balthica community on fluxes of nutrients and dissolved organic carbon across the sedimentwater interface. Journal of Experimental Marine Biology and Ecology 337, 178-189. https://doi.org/10.1016/j.jembe.2006.06.025

Montserrat, F., Van Colen, C., Provoost, P., Milla, M., Ponti, M., Van den Meersche, K., Ysebaert, T., Herman, P.M.J., 2009. Sediment segregation by biodiffusing bivalves. Estuarine, Coastal and Shelf Science 83, 379-391.

1127 Morata, N., Michaud, E., Włodarska-Kowalczuk, M., 2015. Impact of early food input on the 1128 Arctic benthos activities during the polar night. Polar Biology 38, 99-114. https://doi.org/10.1007/s00300-013-1414-5

1130 Morata, N., Renaud, P.E., 2008. Sedimentary pigments in the western Barents Sea: A 1131 reflection of pelagic-benthic coupling? Deep Sea Research Part II: Topical Studies in 1132 Oceanography 55, 2381-2389. https://doi.org/10.1016/j.dsr2.2008.05.004 
1133 Mulsow, S., Landrum, P., Robbins, J., 2002. Biological mixing responses to sublethal

1134 concentrations of DDT in sediments by Heteromastus filiformis using a 137Cs marker

1135 layer technique. Marine Ecology Progress Series 239, 181-191.

1136 https://doi.org/10.3354/meps239181

1137 Näkki, P., Setälä, O., Lehtiniemi, M., 2017. Bioturbation transports secondary microplastics to

1138 deeper layers in soft marine sediments of the northern Baltic Sea. Marine Pollution

1139 Bulletin 119, 255-261. https://doi.org/10.1016/j.marpolbul.2017.03.065

1140 Needham, H.R., Pilditch, C.A., Lohrer, A.M., Thrush, S.F., 2011. Context-Specific

1141 Bioturbation Mediates Changes to Ecosystem Functioning. Ecosystems 14, 1096-1109.

$1142 \quad$ https://doi.org/10.1007/s 10021-011-9468-0

1143 Nogaro, G., Charles, F., de Mendonça, J.B., Mermillod-Blondin, F., Stora, G., François-

1144 Carcaillet, F., 2008. Food supply impacts sediment reworking by Nereis diversicolor.

1145 Hydrobiologia 598, 403-408. https://doi.org/10.1007/s10750-007-9135-9

1146 Olli, K., Wexels Riser, C., Wassmann, P., Ratkova, T., Arashkevich, E., Pasternak, A., 2002.

1147 Seasonal variation in vertical flux of biogenic matter in the marginal ice zone and the

1148 central Barents Sea. Journal of Marine Systems 38, 189-204.

1149 https://doi.org/10.1016/S0924-7963(02)00177-X

1150 Ouellette, D., Desrosiers, G., Gagne, J., Gilbert, F., Poggiale, J., Blier, P., Stora, G., 2004.

1151 Effects of temperature on in vitro sediment reworking processes by a gallery

1152 biodiffusor, the polychaete Neanthes virens. Marine Ecology Progress Series 266, 185-

1153 193. https://doi.org/10.3354/meps266185

1154 Ozhigin,V. K., Ingvaldsen, R. B., Loeng,H., Boitsov, V., Karsakov, A., 2011. Introduction to

1155 the Barents Sea. In The Barents Sea. Ecosystem, resources, management. Half a 1156 century of Russian-Norwegian cooperation, pp. 315-328. Ed. by T. Jakobsen, and V. K.

1157 Ozhigin. Tapir Academic Press, Trondheim. 
1158 Pathirana, I., Knies, J., Felix, M., Mann, U., 2013. Towards an improved organic carbon budget for the Barents Sea shelf, marginal Arctic Ocean. Climate of the Past Discussions 9, 4939-4986. https://doi.org/10.5194/cpd-9-4939-2013

1161 Peeken, I., 2016. The Expedition PS92 of the Research Vessel POLARSTERN to the Arctic Ocean in 2015, Berichte zur Polar und Meeresforschung =Reports on polar and marine research, Bremerhaven, Alfred Wegener Institute for Polar and Marine Research 694 , 153 p. https://doi.org/10.2312/BzPM_0694_2016

Petch, D.A., 1986. Selective deposit-feeding by Lumbrineris cf. latreilli (Polychaeta: Lumbrineridae), with a new method for assessing selectivity by deposit-feeding organisms. Marine Biology 93, 443-448.

1168 Piot, A., Nozais, C., Archambault, P., 2014. Meiofauna affect the macrobenthic biodiversityecosystem functioning relationship. Oikos 123, 203-213. https://doi.org/10.1111/j.1600-0706.2013.00631.x

1171 Queirós, A.M., Birchenough, S.N.R., Bremner, J., Godbold, J.A., Parker, R.E., Romero-

Quintana, C.O., Tang, M., Kristensen, E., 2007. Simultaneous study of particle reworking, irrigation transport and reaction rates in sediment bioturbated by the polychaetes Heteromastus and Marenzelleria. Journal of Experimental Marine Biology and Ecology

1179 Rasmussen, T.L., Thomsen, E., 2014. Brine formation in relation to climate changes and ice 1180 retreat during the last 15,000 years in Storfjorden, Svalbard, $76-78^{\circ} \mathrm{N}$. 1181 Paleoceanography 29, 911-929. https://doi.org/10.1002/2014PA002643

1182 Renaud, P.E., Morata, N., Carroll, M.L., Denisenko, S.G., Reigstad, M., 2008. Pelagic- 
benthic coupling in the western Barents Sea: Processes and time scales. Deep Sea Research Part II: Topical Studies in Oceanography 55, 2372-2380. https://doi.org/10.1016/j.dsr2.2008.05.017

1186

Rosli, N., Leduc, D., Rowden, A.A., Clark, M.R., Probert, P.K., Berkenbusch, K., Neira, C., 2016. Differences in meiofauna communities with sediment depth are greater than habitat effects on the New Zealand continental margin: implications for vulnerability to anthropogenic disturbance. PeerJ 4, e2154. https://doi.org/10.7717/peerj.2154

Rouse, G.W., Pleijel, F., 2001. Polychaetes. Oxford University Press, New York, 354 pp.

Sakshaug, E., 2004. Primary and Secondary Production in the Arctic Seas. In: Stein R., MacDonald R.W. (eds) The Organic Carbon Cycle in the Arctic Ocean. Springer, Berlin, Heidelberg.

Sandnes, J., Forbes, T., Hansen, R., Sandnes, B., 2000. Influence of particle type and faunal activity on mixing of di(2-ethylhexyl)phthalate (DEHP) in natural sediments. Marine Ecology Progress Series. 197, 151-167.

Shick, J.M., 1976. Physiological and behavioral responses to hypoxia and hydrogen sulfide in the infaunal asteroid Ctenodiscus crispatus. Marine Biology 37, 279-289. https://doi.org/10.1007/BF00387613

Shields, M.A., Kedra, M., 2009. A deep burrowing sipunculan of ecological and geochemical importance. Deep Sea Research Part I: Oceanographic Research Papers 56, 2057-2064. https://doi.org/10.1016/j.dsr.2009.07.006

Skarðhamar, J., Svendsen, H., 2010. Short-term hydrographic variability in a stratified Arctic fjord. Geological Society, London, Special Publications 344, 51-60. https://doi.org/10.1144/SP344.5

Skogseth, R., Fer, I., Haugan, P.M., 2005. Dense-water production and overflow from an arctic coastal polynya in Storfjorden, in: Drange, H., Dokken, T., Furevik, T., Gerdes, 
R., Berger, W. (Eds.), Geophysical Monograph Series. American Geophysical Union, Washington, D. C., pp. 73-88. https://doi.org/10.1029/158GM07

1210

Smith, J.N., Schafer, C.T., 1984. Bioturbation processes in continental slope and rise sediments delineated by $\mathrm{Pb}-210$, microfossil and textural indicators. Journal of Marine Research 42, 1117-1145. https://doi.org/10.1357/002224084788520738

Smoła, Z.T., Tatarek, A., Wiktor, J.M., Wiktor, J.M.W., Kubiszyn, A., Węsławski, J.M., 2017. Primary producers and production in Hornsund and Kongsfjorden - comparison of two fjord systems. Polish Polar Research 38, 351-373. https://doi.org/10.1515/popore$\underline{2017-0013}$

Soltwedel, T., Hasemann, C., Vedenin, A., Bergmann, M., Taylor, J., Krauß, F., 2019. Bioturbation rates in the deep Fram Strait: Results from in situ experiments at the arctic LTER observatory HAUSGARTEN. Journal of Experimental Marine Biology and Ecology 511, 1-9. https://doi.org/10.1016/j.jembe.2018.11.001

Søreide, J.E., Falk-Petersen, S., Hegseth, E.N., Hop, H., Carroll, M.L., Hobson, K.A., Blachowiak-Samolyk, K., 2008. Seasonal feeding strategies of Calanus in the highArctic Svalbard region. Deep Sea Research Part II: Topical Studies in Oceanography 55, 2225-2244. https://doi.org/10.1016/j.dsr2.2008.05.024

Søreide, J.E., Hop, H., Falk-Petersen, S., Hegseth, E.N., Carroll, M.L., 2006. Seasonal food web structures and sympagic-pelagic coupling in the European Arctic revealed by stable isotopes and a two-source food web model. Progress in Oceanography 71, 5987. https://doi.org/10.1016/j.pocean.2006.06.001

Stead, R.A., Thompson, R.J., 2006. The influence of an intermittent food supply on the feeding behaviour of Yoldia hyperborea (Bivalvia: Nuculanidae). Journal of Experimental Marine Biology and Ecology 332, 37-48. https://doi.org/10.1016/j.jembe.2005.11.001 
1233 Svendsen, H., Beszczynska-Møller, A., Hagen, J.O., Lefauconnier, B., Tverberg, V., Gerland,

1234

1235

1236

1237

1238

1239

1240

1241

1242

1243

1244

1245

1246

1247

1248

1249

1250

1251

1252

1253

1254

1255

1256

1257

S., Ørb, J.B., Zajaczkowski, M., Azzolini, R., Bruland, O., Wiencke, C., Winther, J.-G., Dallmann, W., 2002. The physical environment of Kongsfjorden-Krossfjorden, an Arctic fjord system in Svalbard. Polar Research 21, 133-166.

Tamelander, T., Reigstad, M., Hop, H., Carroll, M.L., Wassmann, P., 2008. Pelagic and sympagic contribution of organic matter to zooplankton and vertical export in the Barents Sea marginal ice zone. Deep Sea Research Part II: Topical Studies in Oceanography 55, 2330-2339. https://doi.org/10.1016/j.dsr2.2008.05.019

Tamelander, T., Renaud, P.E., Hop, H., Carroll, M.L., Ambrose Jr., W.G., Hobson, K.A., 2006. Trophic relationships and pelagic-benthic coupling during summer in the Barents Sea Marginal Ice Zone revealed by stable carbon and nitrogen isotope measurements. Marine Ecology Progress Series 310, 33-46.

Teal, L., Bulling, M., Parker, E., Solan, M., 2008. Global patterns of bioturbation intensity and mixed depth of marine soft sediments. Aquatic Biology 2, 207-218. https://doi.org/10.3354/ab00052

Van Leeuwe, M.A., Tedesco, L., Arrigo, K.R., Assmy, P., Campbell, K., Meiners, K.M., Rintala, J.-M., Selz, V., Thomas, D.N., Stefels, J., Deming, J.W., 2018. Microalgal community structure and primary production in Arctic and Antarctic sea ice: A synthesis. Elem Sci Anth 6. https://doi.org/10.1525/elementa.267

Vanreusel, A., Fonseca, G., Danovaro, R., Da Silva, M.C., Esteves, A.M., Ferrero, T., Gad, G., Galtsova, V., Gambi, C., Da Fonsêca Genevois, V., Ingels, J., Ingole, B., Lampadariou, N., Merckx, B., Miljutin, D., Miljutina, M., Muthumbi, A., Netto, S., Portnova, D., Radziejewska, T., Raes, M., Tchesunov, A., Vanaverbeke, J., Van Gaever, S., Venekey, V., Bezerra, T.N., Flint, H., Copley, J., Pape, E., Zeppilli, D., Martinez, P.A., Galeron, J., 2010. The contribution of deep-sea macrohabitat heterogeneity to global nematode 
diversity: Nematode diversity and habitat heterogeneity. Marine Ecology 31, 6-20. https://doi.org/10.1111/j.1439-0485.2009.00352.x

1260

1261

1262

1263

1264

1265

1266

1267

1268

1269

1270

1271

1272

1273

1274

1275

1276

1277

1278

1279

1280

1281

1282

Venturini, N., Pires-Vanin, A.M.S., Salhi, M., Bessonart, M., Muniz, P., 2011. Polychaete response to fresh food supply at organically enriched coastal sites: Repercussion on bioturbation potential and trophic structure. Journal of Marine Systems 88, 526-541. https://doi.org/10.1016/j.jmarsys.2011.07.002

Viitasalo-Frösén, S., Laine, A., Lehtiniemi, M., 2009. Habitat modification mediated by motile surface stirrers versus semi-motile burrowers: potential for a positive feedback mechanism in a eutrophied ecosystem. Marine Ecology Progress Series 376, 21-32. https://doi.org/10.3354/meps07788

Vinje, T., 2001. Anomalies and Trends of Sea-Ice Extent and Atmospheric Circulation in the Nordic Seas during the Period 1864-1998. Journal of Climate 14, 255-267. https://doi.org/10.1175/1520-0442(2001)014<0255:AATOSI $>2.0 . C O ; 2$

Vinje, T., 2009. Sea-ice. In Ecosystem Barents Sea, pp. 65-82. Ed. by E. Sakshaug, K. Kovacs, and G. Johnsen. Tapir Academic Press, Trondheim, Norway. 62-82 p.

Winkelmann, D., Knies, J., 2005. Recent distribution and accumulation of organic carbon on the continental margin west off Spitsbergen. Geochemistry, Geophysics, Geosystems 6, 1-22. https://doi.org/10.1029/2005GC000916

Włodarska-Kowalczuk, M., Pawłowska, J., Zajączkowski, M., 2013. Do foraminifera mirror diversity and distribution patterns of macrobenthic fauna in an Arctic glacial fjord? Marine Micropaleontology 103 $30-39$. https://doi.org/10.1016/j.marmicro.2013.07.002

Włodarska-Kowalczuk, M., Pearson, T.H., 2004. Soft-bottom macrobenthic faunal associations and factors affecting species distributions in an Arctic glacial fjord (Kongsfjord, Spitsbergen). Polar Biology 27, 155-167. https://doi.org/10.1007/s00300- 
$\underline{003-0568-y}$

1284

1285

1286

1287

1288

1289

1290

1291

1292

1293

1294

1295

1296

1297

1298

1299

1300

1301

1302

1303

1304

1305

1306

1307

WoRMS Editorial Board, 2019. World Register of Marine Species. Available from http://www.marinespecies.org at VLIZ. Accessed 2019-05-08. https://doi.org/10.14284/170

Zaborska, A., Pempkowiak, J., Papucci, C., 2006. Some Sediment Characteristics and Sedimentation Rates in an Arctic Fjord (Kongsfjorden, Svalbard). Ann. Environ. Prot., 8, 79-96.

Zanzerl, H., Dufour, S.C., 2017. The burrowing behavior of symbiotic and asymbiotic thyasirid bivalves. Journal of Conchology 42, 299-308.

\section{Figures and tables:}

List of tables:

Table 1. Main characteristics of the sampling stations.

Table 2. Bottom water (BW) characteristics for each sampling station: $\mathrm{C}_{\mathrm{org}}, \mathrm{N}_{\mathrm{tot}}, \delta^{13} \mathrm{C}, \delta^{15} \mathrm{~N}$ (in $\%)$ and $\mathrm{C} / \mathrm{N}$ values (mean $\pm \mathrm{SD}, \mathrm{n}=3$ ).

Table 3. Sediment variables for each sampling station: sediment type, $\mathrm{C}_{\mathrm{org}}, \mathrm{N}_{\mathrm{tot}}, \delta^{13} \mathrm{C}, \delta^{15} \mathrm{~N}$, $\mathrm{OM}$ (in \%), C/N, Chl $a\left(\mu \mathrm{g} \mathrm{DW} \mathrm{g}{ }^{-1}\right)$ and $\mathrm{Chl} a /$ Phaeo values (mean $\pm \mathrm{SD}, \mathrm{n}=$ no of cores).

Table 4. Functional traits, relative density and biomass of the three dominant taxa for each sampling station. Class: P - Polychaeta, B - Bivalvia, An - Anthozoa, As - Asteroidea, O Ophiuroidea, S - Sipunculidea. Mobility and feeding groups (M/F) are marked by codes: mobility type (D - Discretely mobile, M - Mobile, S - Sessile) and feeding type (car carnivore, omn - omnivore, sub - subsurface feeder, sur - surface feeder, sus - suspension feeder). Burrowing depth (BT): 1 - surface burrowing, 2 - subsurface burrowing, 3 - deep burrowing. Tubes (T): “+”- I-shaped tube, “-“- no tube. Sediment mixing types (SMix): biodiffusor (B), upward conveyor (UC), gallery diffusor (GD), downward conveyor (DC). 
1308 Table 5. PERMANOVA results for the multivariate descriptors of benthic communities with

1309 significant pair-wise comparisons results for different groups.

1310 Table 6. SIMPER analysis B/D ratio faunal percentage contribution to the average similarity

1311 for different sampling stations groups. Species that contributed more than $5 \%$ are listed.

1312 Table 7. Results of DistLM procedure for fitting environmental variables to the macofauna

1313 community data. \%Var - percentage of explained variance; \%Cum - cumulative percentage

1314 explained by the added variable. Significance level $\mathrm{p}<0.05$. Environmental factors: D -

1315 depth, $\mathrm{S}$ - salinity, T - temperature, types of sediment (mud, sand, gravel), BW $\mathrm{C}_{\text {org }}-$ bottom

1316 water $\mathrm{C}_{\text {org }}$, BW $\mathrm{N}_{\text {tot }}-$ bottom water $\mathrm{N}_{\text {tot }}$, BW $\delta^{13} \mathrm{C}-$ bottom water $\delta^{13} \mathrm{C} \mathrm{BW}, \mathrm{BW} \delta^{15} \mathrm{~N}-$

1317 bottom water $\delta^{15} \mathrm{~N}, \mathrm{BW} \mathrm{C} / \mathrm{N}-$ bottom water $\mathrm{C} / \mathrm{N}$, Sed $\mathrm{C}_{\mathrm{org}}-\mathrm{C}_{\mathrm{org}}$ concentration in sediment,

1318 Sed $\mathrm{N}_{\text {tot }}-$ sediment $\mathrm{N}_{\text {tot }}$, Sed $\delta^{13} \mathrm{C}-$ sediment $\delta^{13} \mathrm{C}$, Sed $\delta^{15} \mathrm{~N}-\operatorname{sediment} \delta^{15} \mathrm{~N}$, Sed $\mathrm{C} / \mathrm{N}-$

1319 sediment C/N, SOM - sediment organic matter, Chl $a$ - sediment Chlorophyll $a$ and Chl

$1320 \quad a /$ Phaeo - sediment Phaeopigments.

1321 Table 8. Spearman's rank correlation analyses among biological and physical parameters.

1322 Significant values are marked in bold $(\mathrm{p}<0.05)$

1323

1324 List of figures:

1325 Fig. 1. Geographical location of the study region (A) and (B) sampling locations during two

1326 cruises (AX - ARCEx, PS - TRANSSIZ) with two major currents surrounding Svalbard:

1327 WSC - West Spitsbergen Current, warm Atlantic waters (black) and the ESC - East

1328 Spitsbergen Current, cold Arctic waters (gray) (after Svendsen et al., 2002).

1329 Fig. 2. Percentages of mobility and feeding groups at different sampling stations. Station ST/8

1330 marked with * was sampled in summer season. Functional traits codes: mobility type (D -

1331 Discretely mobile (yellow), M - Mobile (green), S - Sessile (blue)) and feeding type (car - 
1332 carnivore, omn - omnivore, sub - subsurface feeder, sur - surface feeder, sus - suspension

1333 feeder).

1334 Fig. 3. PCO analysis for macrobenthic communities based on species biomass to density ratio,

1335 and the Bray-Curtis similarity among four sampling areas: A (Hornsund, Van Mijenfjorden);

1336 B (Storfjorden); C (Barents Sea shelf); D (northern Barents Sea and Nansen Basin).

1337 Significantly correlated species with the PCO coordinates $(r>0.5)$ are shown on the plot.

1338 Fig. 4. Mean density (ind. $\left./ \mathrm{m}^{-2}\right)(\mathrm{A})$ and biomass $\left(\mathrm{g} / \mathrm{m}^{-2}\right)(\mathrm{B}) ; \pm \mathrm{SE}, \mathrm{n}=$ no of cores (Table 1$)$ at 1339 stations sampled in Van Mijenfjorden, Hornsund (group A); Storfjorden (group B); Barents

1340 Sea shelf (group C); northern Barents Sea and Nansen Basin (group D). Station ST/8 marked 1341 with * was sampled in summer season. Kruskal - Wallis results for differences between 1342 sampling sites are given; significant test results are marked with ** $(\mathrm{p}<0.05)$.

1343 Fig. 5. Distance-based Redundancy Analysis (dbRDA) plot of the DistLM model visualizing 1344 the relationships between the environmental parameters and the biomass/density ratio of 1345 species between four sampling areas: A (Hornsund, Van Mijenfjorden); B (Storfjorden); C 1346 (Barents Sea shelf); D (northern Barents Sea and Nansen Basin). Environmental variables 1347 with Pearson rank correlations with dbRDA axes $>0.5$ are shown. Environmental factors: D 1348 depth, $\mathrm{S}$ - salinity, $\mathrm{T}$ - temperature, types of sediment (mud, sand, gravel), BW $\mathrm{C}_{\text {org }}$ - bottom 1349 water $\mathrm{C}_{\text {org }}$, BW $\mathrm{N}_{\text {tot }}-$ bottom water $\mathrm{N}_{\text {tot }}, \mathrm{BW} \delta^{15} \mathrm{~N}-$ bottom water $\delta^{15} \mathrm{~N}, \mathrm{BW} \mathrm{C} / \mathrm{N}-$ bottom 1350 water $\mathrm{C} / \mathrm{N}$, Sed $\mathrm{C}_{\text {org }}-\mathrm{C}_{\text {org }}$ concentration in sediment, Sed $\delta^{13} \mathrm{C}-$ sediment $\delta^{13} \mathrm{C}$, Sed $\mathrm{C} / \mathrm{N}-$ 1351 sediment $\mathrm{C} / \mathrm{N}, \mathrm{Chl} a$-sediment Chlorophyll $a$ and Chl $a / \mathrm{Phaeo}$ - sediment Phaeopigments.

1352 Fig. 6. Mean bioturbation coefficients: Db - biodiffusion $\left(\mathrm{cm}^{-2} \mathrm{y}^{-1}\right)(A)$ and $\mathrm{r}-$ non-local $\left(\mathrm{y}^{-1}\right)$ 1353 (B); $\pm \mathrm{SE}, \mathrm{n}=$ no of cores (Table 1) at stations sampled in Van Mijenfjorden, Hornsund (group 1354 A); Storfjorden (group B); Barents Sea shelf (group C); northern Barents Sea and Nansen 1355 Basin (group D). Station ST/8 marked with * was sampled in summer season. Kruskal - 
1356 Wallis results for differences between sampling sites are given; significant test results are 1357 marked with $* *(\mathrm{p}<0.05)$. 


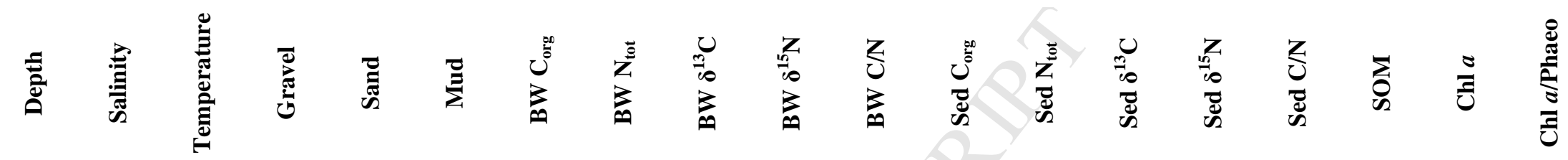

\begin{tabular}{|c|c|c|c|c|c|c|c|c|c|c|c|c|c|c|c|c|c|c|c|}
\hline Depth & - & 0.8 & 0.8 & 0.7 & -0.2 & -0.0 & -0.1 & 0.3 & 0.1 & -0.5 & -0.6 & -0.6 & -0.3 & 0.4 & -0.2 & -0.5 & -0.4 & -0.2 & -0.5 \\
\hline Salinity & 0.8 & - & 0.7 & 0.3 & -0.4 & 0.4 & 0.2 & 0.3 & -0.0 & -0.3 & -0.3 & -0.2 & 0.3 & 0.8 & -0.1 & -0.5 & 0.1 & 0.2 & -0.3 \\
\hline Temperature & 0.8 & 0.7 & - & 0.6 & -0.3 & -0.0 & -0.0 & 0.1 & -0.2 & -0.2 & -0.3 & -0.3 & -0.2 & 0.5 & 0.0 & -0.3 & -0.2 & -0.1 & -0.5 \\
\hline Gravel & 0.7 & 0.3 & 0.6 & - & -0.2 & -0.0 & -0.1 & 0.2 & 0.3 & -0.5 & -0.6 & -0.7 & -0.5 & -0.0 & -0.3 & -0.5 & -0.7 & -0.4 & -0.4 \\
\hline Sand & -0.2 & -0.4 & -0.3 & -0.2 & - & -0.9 & -0.8 & -0.7 & -0.6 & -0.1 & 0.2 & -0.2 & -0.5 & -0.5 & 0.5 & 0.2 & -0.2 & -0.3 & 0.0 \\
\hline Mud & -0.0 & 0.4 & -0.0 & -0.0 & -0.9 & - & 0.7 & 0.6 & 0.6 & 0.2 & 0.0 & 0.3 & 0.7 & 0.5 & -0.4 & -0.3 & 0.4 & 0.5 & 0.1 \\
\hline BW $\mathbf{C}_{\text {org }}$ & -0.1 & 0.2 & -0.0 & -0.1 & -0.8 & 0.7 & - & 0.8 & 0.6 & 0.1 & -0.2 & 0.4 & 0.4 & 0.4 & -0.7 & 0.2 & 0.3 & 0.4 & 0.3 \\
\hline BW $\mathbf{N}_{\text {tot }}$ & 0.3 & 0.3 & 0.1 & 0.2 & -0.7 & 0.6 & 0.8 & - & 0.7 & -0.4 & -0.7 & -0.1 & 0.1 & 0.4 & -0.8 & -0.1 & 0.1 & 0.4 & 0.4 \\
\hline$B W \delta^{13} C$ & 0.1 & -0.0 & -0.2 & 0.3 & -0.6 & 0.6 & 0.6 & 0.7 & - & -0.1 & -0.4 & -0.1 & 0.1 & -0.1 & -0.5 & -0.2 & -0.1 & 0.0 & 0.1 \\
\hline$B W \delta^{15} \mathbf{N}$ & -0.5 & -0.3 & -0.2 & -0.5 & -0.1 & 0.2 & 0.1 & -0.4 & -0.1 & - & 0.8 & 0.8 & 0.5 & -0.2 & 0.5 & 0.5 & 0.5 & -0.2 & -0.3 \\
\hline BW C/N & -0.6 & -0.3 & -0.3 & -0.6 & 0.2 & 0.0 & -0.2 & -0.7 & -0.4 & 0.8 & - & 0.7 & 0.5 & -0.1 & 0.6 & 0.3 & 0.4 & -0.0 & -0.1 \\
\hline Sed $C_{\text {org }}$ & -0.6 & -0.2 & -0.3 & -0.7 & -0.2 & 0.3 & 0.4 & -0.1 & -0.1 & 0.8 & 0.7 & - & 0.7 & 0.1 & 0.3 & 0.6 & 0.8 & 0.1 & 0.0 \\
\hline Sed $N_{\text {tot }}$ & -0.3 & 0.3 & -0.2 & -0.5 & -0.5 & 0.7 & 0.4 & 0.1 & 0.1 & 0.5 & 0.5 & 0.7 & - & 0.5 & 0.2 & -0.1 & 0.8 & 0.5 & 0.0 \\
\hline $\operatorname{Sed} \delta^{13} C$ & 0.4 & 0.8 & 0.5 & -0.0 & -0.5 & 0.5 & 0.4 & 0.4 & -0.1 & -0.2 & -0.1 & 0.1 & 0.5 & - & -0.2 & -0.5 & 0.3 & 0.7 & 0.1 \\
\hline $\operatorname{Sed} \delta^{15} N$ & -0.2 & -0.1 & 0.0 & -0.3 & 0.5 & -0.4 & -0.7 & -0.8 & -0.5 & 0.5 & 0.6 & 0.3 & 0.2 & -0.2 & - & 0.2 & 0.3 & -0.4 & -0.6 \\
\hline Sed C/N & -0.5 & -0.5 & -0.3 & -0.5 & 0.2 & -0.3 & 0.2 & -0.1 & -0.2 & 0.5 & 0.3 & 0.6 & -0.1 & -0.5 & 0.2 & - & 0.3 & -0.4 & 0.0 \\
\hline SOM & -0.4 & 0.1 & -0.2 & -0.7 & -0.2 & 0.4 & 0.3 & 0.1 & -0.1 & 0.5 & 0.4 & 0.8 & 0.8 & 0.3 & 0.3 & 0.3 & - & 0.3 & -0.0 \\
\hline Chl $a$ & -0.2 & 0.2 & -0.1 & -0.4 & -0.3 & 0.5 & 0.4 & 0.4 & 0.0 & -0.2 & -0.0 & 0.1 & 0.5 & 0.7 & -0.4 & -0.4 & 0.3 & - & 0.7 \\
\hline Chl $a$ /Phaeo & -0.5 & -0.3 & -0.5 & -0.4 & 0.0 & 0.1 & 0.3 & 0.4 & 0.1 & -0.3 & -0.1 & 0.0 & 0.0 & 0.1 & -0.6 & 0.0 & -0.0 & 0.7 & - \\
\hline
\end{tabular}


- This is the first complex report on bioturbation in spring to summer transition conducted over a large depth gradient in the Arctic Ocean.

- Benthic community structure and related biodiffusion and non-local transport varied in Svalbard fjords, Barents Sea and Nansen Basin.

- Changes in environmental conditions, and related changes in quality and quantity of available organic matter, had impact on benthic communities and bioturbation.

- Large inputs of fresh OM to the seabed can trigger bioturbation activities. 